\title{
Comparative study of three commonly used continuous deterministic methods for modeling gene regulation networks
}

Martin T Swain², Johannes J Mandel ${ }^{1}$, Werner Dubitzky ${ }^{2 *}$

\begin{abstract}
Background: A gene-regulatory network (GRN) refers to DNA segments that interact through their RNA and protein products and thereby govern the rates at which genes are transcribed. Creating accurate dynamic models of GRNs is gaining importance in biomedical research and development. To improve our understanding of continuous deterministic modeling methods employed to construct dynamic GRN models, we have carried out a comprehensive comparative study of three commonly used systems of ordinary differential equations: The S-system (SS), artificial neural networks (ANNs), and the general rate law of transcription (GRLOT) method. These were thoroughly evaluated in terms of their ability to replicate the reference models' regulatory structure and dynamic gene expression behavior under varying conditions.

Results: While the ANN and GRLOT methods appeared to produce robust models even when the model parameters deviated considerably from those of the reference models, SS-based models exhibited a notable loss of performance even when the parameters of the reverse-engineered models corresponded closely to those of the reference models: this is due to the high number of power terms in the SS-method, and the manner in which they are combined. In cross-method reverse-engineering experiments the different characteristics, biases and idiosynchracies of the methods were revealed. Based on limited training data, with only one experimental condition, all methods produced dynamic models that were able to reproduce the training data accurately. However, an accurate reproduction of regulatory network features was only possible with training data originating from multiple experiments under varying conditions.

Conclusions: The studied GRN modeling methods produced dynamic GRN models exhibiting marked differences in their ability to replicate the reference models' structure and behavior. Our results suggest that care should be taking when a method is chosen for a particular application. In particular, reliance on only a single method might unduly bias the results.
\end{abstract}

\section{Background}

Regulation of gene expression (or gene regulation) refers to processes that cells use to create functional gene products (RNA, proteins) from the information stored in genes (DNA). These processes range from DNA-RNA transcription to the post-translational modification of proteins. Gene regulation is essential for life as it increases the versatility and adaptability of an organism by allowing it to express protein when needed. While

\footnotetext{
* Correspondence: w.dubitzky@ulster.ac.uk

2University of Ulster, School of Biomedical Sciences, Cromore Road, Coleraine BT52 1SA, Co. Londonderry, UK

Full list of author information is available at the end of the article
}

aspects of gene regulation are well understood, many open research questions still remain [1]. Due to the wide availability of well-characterized components from biological gene networks, the stage has been set for mathematical modeling and computational simulation of gene regulatory networks (GRNs). The modeling of biomedical phenomena is inspired by the approach taken in physics. In physics, models (theories) are frequently constructed to explain existing data, then predictions are made, which again are compared to new data. If a sufficient correspondence exists, it is claimed that the phenomenon has been understood. A model should not be a black box, but should be interpretable in some way.
C Biomed Central

() 2010 Swain et al; licensee BioMed Central Ltd. This is an Open Access article distributed under the terms of the Creative Commons Attribution License (http://creativecommons.org/licenses/by/2.0), which permits unrestricted use, distribution, and reproduction in any medium, provided the original work is properly cited. 
Ideally, the model components and elements should have an interpretation in the real world consistent with the existing knowledge. At the same time a model involves a simplification of the real world. The ability to construct, analyze, and interpret qualitative and quantitative GRN models is becoming increasingly important tool in studying gene regulation [2]. Because of its potential to help improve our understanding of gene regulation, modeling and simulation of GRNs has received considerable interest in the bioinformatics and computational biology communities. Many mathematical and computational techniques have been proposed in recent years. In practice, different mathematical techniques generate models with different properties and features. Therefore, it is very important for users to understand the merits and limitations of these approaches.

The dynamic behavior and regulatory interactions of genes can be revealed by time-series experiments, that is, experiments that measure the expression of multiple genes over time [3-6]. As this type of experimental data becomes more readily available, mathematical modeling and computational simulation become an important tool for investigating the structure and time-dependent behavior of GRNs. In contrast to static gene expression data, the modeling and simulation approach allows the determination of stable states in response to a condition or stimulus as well as the identification of pathways and networks that are activated in the process [7]. Besides logical [8-10] and stochastic [11] modeling approaches, various continuous modeling methods capable of capturing such complex behavior deterministically are commonly used. A range of mathematical methods facilitating the reverse-engineering of quantitative, dynamic GRN models from time-series expression data have been reported in the literature [12].

Our study focused primarily on continuous deterministic simulation methods used to model and simulate GRNs on the basis of time-variant gene expression data. The approaches considered in our study concentrate on GRN abstractions that ignore intricate intermediate biological processes of cellular gene regulation, such as splicing, capping, translation, binding and unbinding [13]. Because of their importance in the field and their common use, we have compared the following three mathematical methods:

- The S-system (SS) method [14];

- The artificial neural network (ANN) method [15];

- The general rate law of transcription (GRLOT) method [16].

All three methods describe dynamic systems by nonlinear equations, yet they are different in the way they process regulatory signals and, hence, in how they model gene expression and gene regulation. In addition, these methods employ different model parameters to represent gene-regulatory mechanisms. Thus, each method conveys a different conceptual view of the underlying GRN. Our comparison of the three methods was guided by the following questions:

1. How do the methods fare in terms of their ability to estimate model parameters from dynamic gene expression data?

2. How do the methods fare in terms of their ability to accurately predict dynamic gene expression data?

3. To what extent are the methods interchangeable? 4. Are there any inherent biases or limitations in the individual methods?

To answer the above questions we developed artificial data sets based on a number of model networks that were selected because they either incorporate motifs frequently seen in GRNs, or because they have been specifically discussed in the literature in the context of the three mathematical methods of interest. The advantages of using artificial data sets is due to the ease, speed and flexibility with which different experiments can be performed and evaluated, allowing validation of the methods under a range of tightly controlled conditions which would be very difficult, expensive and time-consuming to perform with real biological data. However, computer generated data sets feature important differences when compared to real biological data $[17,18]$, and so we have also included a recent biological data set generated by Cantone et al. specifically for in vivo assessment of reverse-engineering and gene network modelling approaches [19]. The reverse-engineering experiments we have performed on this biological network play an important role in demonstrating that the results we have obtained from our model networks are applicable to real biological networks.

Our study is relatively comprehensive and involved. To facilitate a clear understanding of our study design, we outline the main logical steps below. The diagram in Figure 1 summarizes the workflow of our experimental set-up. More detail of the data, models and results corresponding to the steps in the study is provided in the main body of the paper, in Section 2 and Section 4.

1. To investigate a range of different conditions, we obtain two time-series data sets for a biological network from the literature [19], and we define and construct five artificial network models and set their parameters and initial expression levels.

2. We wish to explore how the quantity of available data influences the models produced by reverse- 


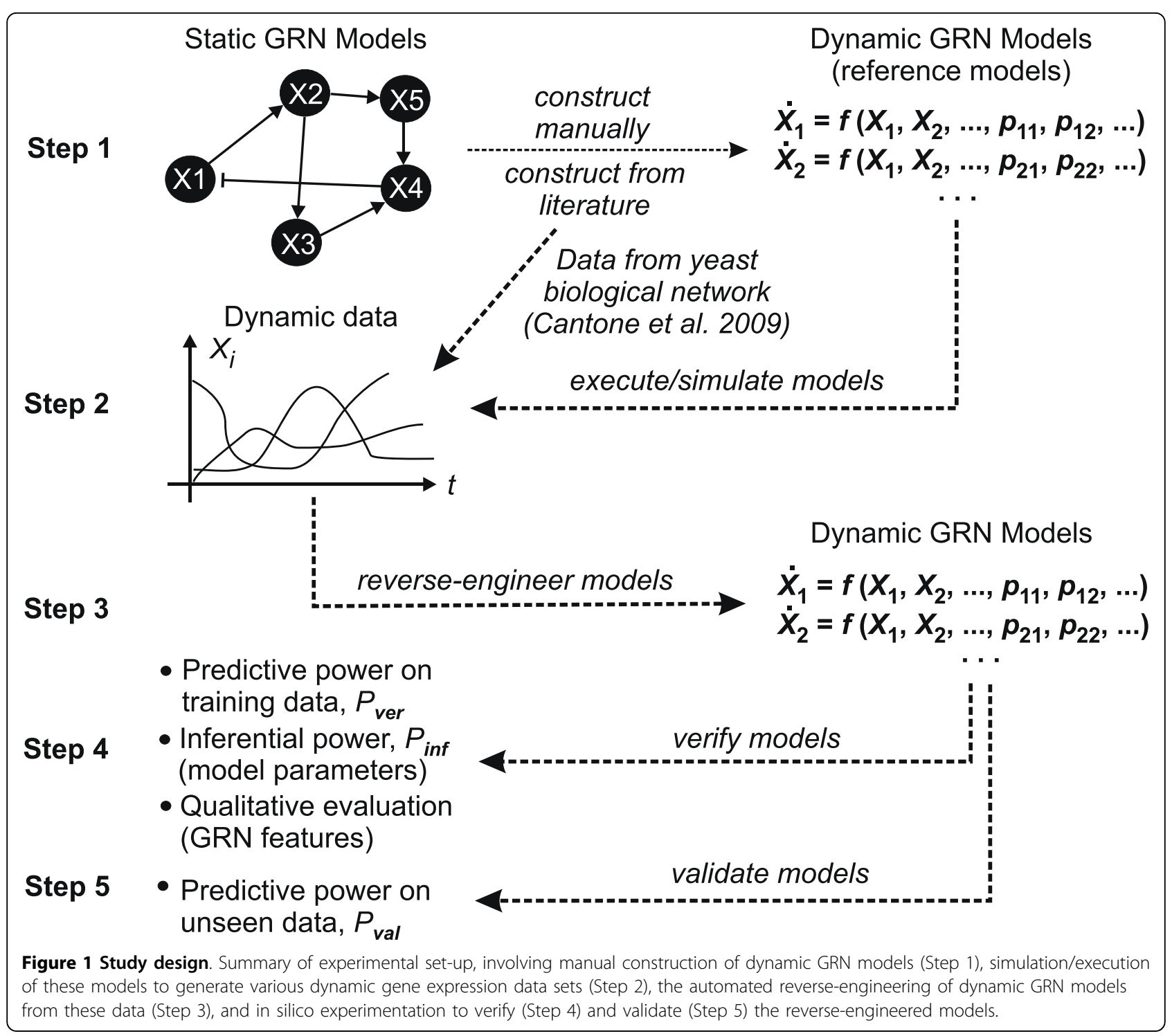

engineering. Therefore, for each artificial network model we generated two sets of data -sparse and detailed data - using each mathematical method. Sparse data is based on a single model simulation, detailed data is based on five simulations each with a different set of initial condition.

3. Here we perform the reverse-engineering of dynamic GRN models from the artificial and biological data sets using an evolutionary algorithm [20]. In order to highlight differences and idiosynchracies between and within the models, we apply each mathematical method to all the available data sets. A large number of models are generated in this step, investigating combinations of different data sets, experimental scenarios, and mathematical methods. 4. This step is designed to assess specific aspects of the models, and to elucidate the extent to which the reverse-engineered models are able to discover the original GRN models and their parameters, or to reproduce their dynamic behavior according to the relevant biological or artificial time-series data sets. 5. The final step is designed to investigate if the GRN models are able to predict how the network will behave under new conditions, and if the mathematical methods are all able to correctly capture the behavior of the GRNs: if this is true then all our reverse-engineered models should behave in a similar fashion under new conditions and make similar predictions about the network behavior.

This paper is structured as follows: first we outline the mathematical methods, then we discuss their different characteristics, strengths and weaknesses, followed by the different GRN models to which we apply them. We 
then outline our results and relate them to the experimental workflow. Before concluding we give a detailed discussion of differences between the mathematical methods as shown by our results.

\subsection{GRN modeling methods}

The non-linear differential equations of the three modeling methods investigated in this study describe the mutual activating and repressing influences of genes in a GRN at a high-level of abstraction. In particular, it is assumed that the rate of gene expression depends exclusively on the concentration of gene products arising from the nodes (genes) of the GRN. This means that the influence of other molecules (e.g., transcription factors) and cellular processes (translation) is not taken into account directly. Even with these limitations, dynamic GRN models of this kind can be useful in deciphering basic aspects of gene-regulatory interactions. The three methods we have studied have been widely used to model dynamic GRNs. One major advantage of all three methods lies in their simple homogeneous structures, as this allows the settings of parameter discovering software to be easily customized for these structures. In addition, all three modeling methods either already have the potential to describe additional levels of detail, or their structures can be easily extended for this purpose.

The three methods describe dynamic GRN models by means of a system (or set) of ordinary differential equations. For a GRN comprising $N$ genes, $N$ differential equations are used to describe the dynamics of $N$ gene product concentrations, $X_{i}$ with $i=1, \ldots, N$. In all three methods, the expression rate $d X_{i} / d t$ of a gene product concentration may depend on the expression level of one or more gene products of the genes $X_{j}$, with $j=1$, ..., $N$. Thus, the gene product concentration $X_{i}$ may be governed by a self-regulatory mechanism (when $i=j$ ), or it may be regulated by products of other genes in the GRN. The three modeling methods differ in the way they represent and calculate expression rates. Before discussing such differences, we introduce the three modeling methods in some detail.

\subsubsection{The artificial neural network (ANN) method}

Vohradsky [15] introduced ANNs as a modeling method capable of describing the dynamic behavior of GRNs. The way this method represents and calculates expression rates depends on the weighted sum of multiple regulatory inputs. This additive input processing is capable of representing logical disjunctions. The expression rate is restricted to a certain interval where a sigmoidal transformation maps the regulatory input to the expression interval. ANNs provide an additional external input which has an influence on this transformation in that it can regulate the sensitivity to the summed regulatory input. Finally, the ANN method defines the degradation of a gene product on the basis of standard mass-action kinetics.

Formally, the ANN method is defined as:

$$
\frac{d X_{i}}{d t}=v_{i} \cdot f\left(\sum_{j=1}^{N} w_{i j} X_{j}-\vartheta_{i}\right)-k_{i} X_{i} \quad \vartheta_{i} v_{i} k_{i}>0
$$

The parameters of the ANN method have the following biological interpretations:

$N$ : Number of genes in the GRN to be modeled. The genes of the GRN are indexed by $i$ and $j$, where $i, j$ $=1, \ldots, N$.

$v_{i}$ : Maximal expression rate of gene $i$.

$w_{i j}$ : The connection weight or strength of control of gene $j$ on gene $i$. Positive values of $w_{i j}$ indicate activating influences while negative values define repressing influences.

$\vartheta_{i}$ : Influence of external input on gene $i$, which modulates the gene's sensitivity of response to activating or repressing influences.

$f$ : Represents a non-linear sigmoid transfer function modifying the influence of gene expression products $X_{j}$ and external input $\vartheta_{i}$ to keep the activation from growing without bounds.

$k_{i}$ : Degradation of the $i$-th gene expression product.

The mathematical properties of the ANN method have been well studied because it is a special case of a recurrent neural network [15]. In particular, the symmetry of the matrix of connection weights $w_{i j}$ influences whether the network dynamics are oscillatory or whether they converge on a steady (or even chaotic) state. High positive or negative values of the external input, $\vartheta_{i}$, reduce the effect of the connection weights. This is explored in Case D where $\vartheta_{i}$ has been interpreted as a delay to the reaction kinetics of the transcriptional machinery [15].

\subsubsection{The S-system (SS) method}

Savageau [14] proposed the synergistic system or S-system (SS) as a method to model molecular networks. When modeling GRNs with the SS method, the expression rates are described by the difference of two products of power-law functions, where the first represents the activation term and the second the degradation term of a gene product $X_{i}$. This multiplicative input processing can be used to define logical conjunctions for both the regulation of gene expression processes and for the regulation of degradation processes. The SS method has no restrictions in the gene expression rates and thus does not implicitly describe saturation. 
Formally, the SS method is defined as:

$$
\frac{d X_{i}}{d t}=\alpha_{i} \prod_{j=1}^{N} X_{j}^{g_{i j}}-\beta_{i} \prod_{j=1}^{N} X_{j}^{h_{i j}} \quad \alpha_{i}, \beta_{i}>0, \quad g_{i j}, h_{i j} \in \Re .
$$

The parameters of the SS method have the following biological interpretations:

$N$ : Number of genes in the GRN to be modeled. The genes of the GRN are indexed by $i$ and $j$, where $i, j$ $=1, \ldots, N$.

$\alpha_{i}$ : Rate constant of activation term; in SS GRN models, all activation (up-regulation) processes of a gene $i$ are aggregated into a single activation term.

$\beta_{i}$ : Rate constant of degradation term; in SS GRN models, all degradation processes of a gene $i$ are aggregated into a single degradation term.

$g_{i j}, h_{i j}$ : Exponential parameters called kinetic order. These parameters describe the interactive influences of gene $j$ on gene $i$. Positive values of $g_{i j}$ indicate an activating influence on the expression of gene $i$, whereas inhibiting influences are represented by negative values. Similarly, positive values of $h_{i j}$ indicate increasing degradation of the gene product $X_{i}$, whereas decreasing degradation is represented by negative values.

The parameters used in SS models have a clear physical meaning and can be measured experimentally [21], yet they describe phenomenological influences, as opposed to stoichiometric rate constants in general mass action (GMA) systems [22]. The SS method generalizes mass-action kinetics by aggregating all individual processes into a single activation and a single degradation term (per gene). In contrast, the GMA system defines all individual processes $k$ with $k=1, \ldots, R$ with the sum of power-law functions [23] according to:

$$
\begin{aligned}
& \frac{d X_{i}}{d t}=\sum_{k=1}^{R} \alpha_{i k} \prod_{j=1}^{N} X_{j}^{g_{i j k}}-\sum_{k=1}^{R} \beta_{i k} \prod_{j=1}^{N} X_{j}^{h_{i j k}} \quad \alpha_{i k}, \beta_{i k}>0, \\
& g_{i j k}, h_{i j k} \in \Re .
\end{aligned}
$$

The parameters of the GMA system have the following biological interpretations:

$\alpha_{i}$ : Rate constant of activation process $k$.

$\beta_{i k}$ : Rate constant of degradation process $k$.

$g_{i j k}$ : Exponential parameter called kinetic order describing the interactive influence of $X_{j}$ on gene $i$ of process $k$.

$h_{i j k}$ : Exponential parameter called kinetic order describing the interactive influence of $X_{j}$ on gene $i$ of process $k$.
1.1.3 The general rate law of transcription (GRLOT) method The GRLOT method has been used to generate benchmark time-series data sets to facilitate the evaluation of different reverse-engineering approaches [16,24]. GRLOT models multiply individual regulatory inputs. Activation and inhibition are represented by different functional expressions that are similar to Hill kinetics, which allow the inclusion of cooperative binding events [16]. Identical to the ANN, the degradation of gene products is defined via mass-action kinetics.

Formally, the GRLOT method is defined as:

$$
\begin{aligned}
& \frac{d X_{i}}{d t}=v_{i} \prod_{j}\left(\frac{K i_{j}^{n_{j}}}{I_{j}^{n_{j}}+K i_{j}^{n_{j}}}\right) \times \prod_{k}\left(\frac{A_{k}^{n_{k}}}{A_{k}^{n_{k}}+K a_{k}^{n_{k}}}\right)-k_{i} X_{i} \\
& v_{i}, K i_{j}, K a_{j}, k_{i}>0,
\end{aligned}
$$

The parameters of the GRLOT method have the following biological interpretations:

$v_{i}$ : Maximal expression rate of gene $i$.

$I_{j}$ : Inhibitor (repressor) $j$.

$A_{k}$ : Activator $k$; the number of inhibitors $I$, and the number of activators $A$ can be related to the total number of genes by $I+A \leq N$.

$K i_{j}$ : Concentration at which the influence of inhibitor $j$ is half of its saturation value.

$K a_{k}$ : Concentration at which the influence of activator $k$ is half of its saturation value.

$n_{j}, n_{k}$ : Regulate the sigmoidicity of the interaction behavior in the same way as Hill coefficients in enzyme kinetics.

$k_{i}$ : Degradation of the $i$-th gene expression product.

\subsection{Systematic biases in the ANN, SS and GRLOT methods} Major differences among the three methods for GRN modeling can be found in the limitation of expression rates, regulation of degradation processes, in the processing of multiple inputs, and in the interpretation of model parameters.

\section{Limitation of expression rates}

The SS method has no restrictions on expression rates, $d X_{i} / d t$, while the ANN and GRLOT methods restrict expression rates to the interval $\left[0, v_{i}\right]$ through a sigmoid function. The three methods have in common that concentration changes are not synchronized, that is, the formation or synthesis of a molecule does not necessarily entail the reduction or degradation of other molecules. (In particular, this can quickly lead to unrealistic dynamics in SS models as singularities may arise when modeling inhibition and the inhibitor reaches a near-zero concentration.) However, the expression concentration of 
a gene product must reach a saturation level at some point and a sigmoid expression rate response is thought to act as the molecular switch that models this behavior [25].

\section{Regulation of degradation processes}

The SS method covers a wider spectrum of regulatory targets since SS models are able to describe the regulation of both transcription and degradation processes.

\section{Multiple input processing}

GRN models based on the ANN method weight and sum multiple regulatory inputs. This contrasts with dynamic GRN models based on the SS and GRLOT methods, where regulatory inputs are exponentiated and multiplied. The input processing in SS and GRLOT models certainly accord better with the fundamentals of reaction kinetics and collision theory (chemical reaction rates correlate with the multiplied concentrations of the reactants) than ANN models. Nevertheless, none of the three methods follow strictly the chemical reaction processes as a single kinetic equation typically comprises several processes. Instead, all methods are designed to be flexible enough to find phenomenological expressions for their approximations of experimentally observed behavior [15,22]. In particular, for the SS method, we have found that the unrestricted expression rates coupled with multiple input processing based on multiplied exponentials is a serious flaw, frequently leading to extreme sensitivity and unstable dynamics (as we describe in Section 2).

\section{Parameter interpretation}

The different parameters used in the three methods describe activating (inducing) and inhibiting (repressing) interactions among the genes of a GRN:

- In the ANN method, the parameters $w_{i j}$ can be intuitively used to define activation and inhibition by assigning positive or negative values, respectively. Here, small absolute values indicate a minor impact on the transcription process (or a missing regulatory interaction) and large absolute values indicate a correspondingly major impact on the transcription process. However, one should be aware that the multiple regulatory inputs required in the case of co-regulation can compensate each other due to their additive input processing.

- The GRLOT method uses two inverse functional forms to describe activating and inhibiting influences both of which involve two parameters. For example, in the case of activation, each individual dependency is described with a Hill equation where the Hill exponent $n_{i j}$ determines the sigmoid response curve to varying concentration levels of the regulating molecule. Furthermore, the second parameter, $K a_{k}$, allows the concentration of the regulator molecule to be specified, which gives half of its maximal influence on the expression rate. Consequently, high exponent values together with low $K a_{k}$ values, defined for a regulator molecule, indicate that already low concentration levels have a strong activating influence on the transcription process while, on the other hand, low exponent values in combination with high $K a_{k}$ values require high concentration levels of the regulator molecule for effective activation.

- The behavior of SS models is mainly determined by the exponent values $g_{i j}$ and $h_{i j}$. Similar to the ANN method, high absolute values define strong influence, whereas small absolute values indicate weak influence. However, the dynamics can become particularly complicated when describing inhibiting dependencies using negative exponent values. Here, the effect depends strongly on the actual concentration ranges of the inhibitor which can introduce singular behavior at near-zero concentrations.

These differences make each method unique in its application to modeling GRNs. Table 1 provides a summary of the characteristics of the three modeling methods.

\subsection{Manually constructing case study models}

Due to the limited number of time points at which gene expression measurements are typically made, the reverse-engineering of GRNs usually constitutes an under-determined problem. Essentially, this means that there are more parameters to estimate than there are measurements [26,27]. Although hundreds of gene activities can be measured simultaneously with microarray experiments, the number of (time-dependent) data points established for each gene is typically small, leading to highly under-determined systems. As a consequence, multiple solutions can be found through reverse-engineering which are able to fit the available data very well, yet which are very weak in their ability to predict dynamic activity under different conditions to those initially explored [28].

Therefore, in order to reduce the number of parameters when performing a modeling study, a first step may involve analyzing gene expression profiles and performing clustering of genes exhibiting similar dynamics [29]. However, the relationships between co-regulated genes are very complex [30] and it is important to identify the key genes that act as regulators to the clusters of co-expressed genes [31]. Once the gene regulators are identified they may be used to construct simplified network models that are useful for reverse-engineering [32]. For instance, Kimura and colleagues [33] built a quantitative model comprising 24 gene groups found in 
Table 1 Method characteristics

\begin{tabular}{llll}
\hline Characteristic & ANN & SS & GRLOT \\
\hline number of parameters & $N^{*}(N+3)$ & $N^{*}(2 * N+2)$ & yes \\
\hline $\begin{array}{l}\text { limitation (saturation and } \\
\text { sigmoidicity) of expression rates }\end{array}$ & yes & no & additive, logical \\
\hline $\begin{array}{l}\text { Processing of multiple signals } \\
\text { disjunction (OR) }\end{array}$ & $\begin{array}{l}\text { multiplicative, logical conjunction (AND) } \\
\text { corresponds well with collision theory }\end{array}$ & $\begin{array}{l}\text { multiplicative, logical conjunction (AND) } \\
\text { corresponds well with collision theory }\end{array}$ \\
\hline $\begin{array}{l}\text { regulation of degradation } \\
\text { processes }\end{array}$ & no yes & no \\
\hline singularities & no & yes & no \\
\hline
\end{tabular}

Characteristics of the artificial neural network (ANN), the S-system (SS) and the General Rate Law Of Transcription (GRLOT) methods.

612 putative open reading frames measured with UvrA gene disruptant experiments in Thermus thermophilus HB8 strains, while Guthke and co-workers [34] clustered a total of 1336 genes into six clusters for dynamic network reconstruction from gene expression data. The latter example was used to investigate the immune response during bacterial infection of peripheral blood mononuclear cells.

With all three mathematical systems introduced in Section 1.1, we construct a set of case study models comprising only a small number of genes similar to the gene clusters used in many modeling studies (Figure 2). Furthermore, they describe network motifs comprising regulatory cascades, single input modules, multiple input modules and multi-component loops $[35,36]$. By first manually constructing and simulating these GRNs, and then performing reverse-engineering on the simulated data, we can greatly limit the problems associated with the poor information content of under-sampled data. The case study models are shown in Figure 2.

\subsubsection{Case studies $A, B$ and $C$}

Each of these three cases is modeled using the ANN, SS and GRLOT method. The parameters of each model are manually chosen, though we ensured that the models produce plausible dynamics.

In all models describing the cases A, B and $\mathrm{C}$, we circumscribed regulatory operations to expression processes since the regulation of degradation processes can be only described with the SS method. For each gene product $X_{i}$ we therefore set in all constructed models the degradation rates $k_{i}\left(\beta_{i}\right.$ in the SS method) to 0.3. In all SS models we set the exponent values $h_{i j}$ to 1 . This allows us to concentrate on the transcriptional part of the different methods.

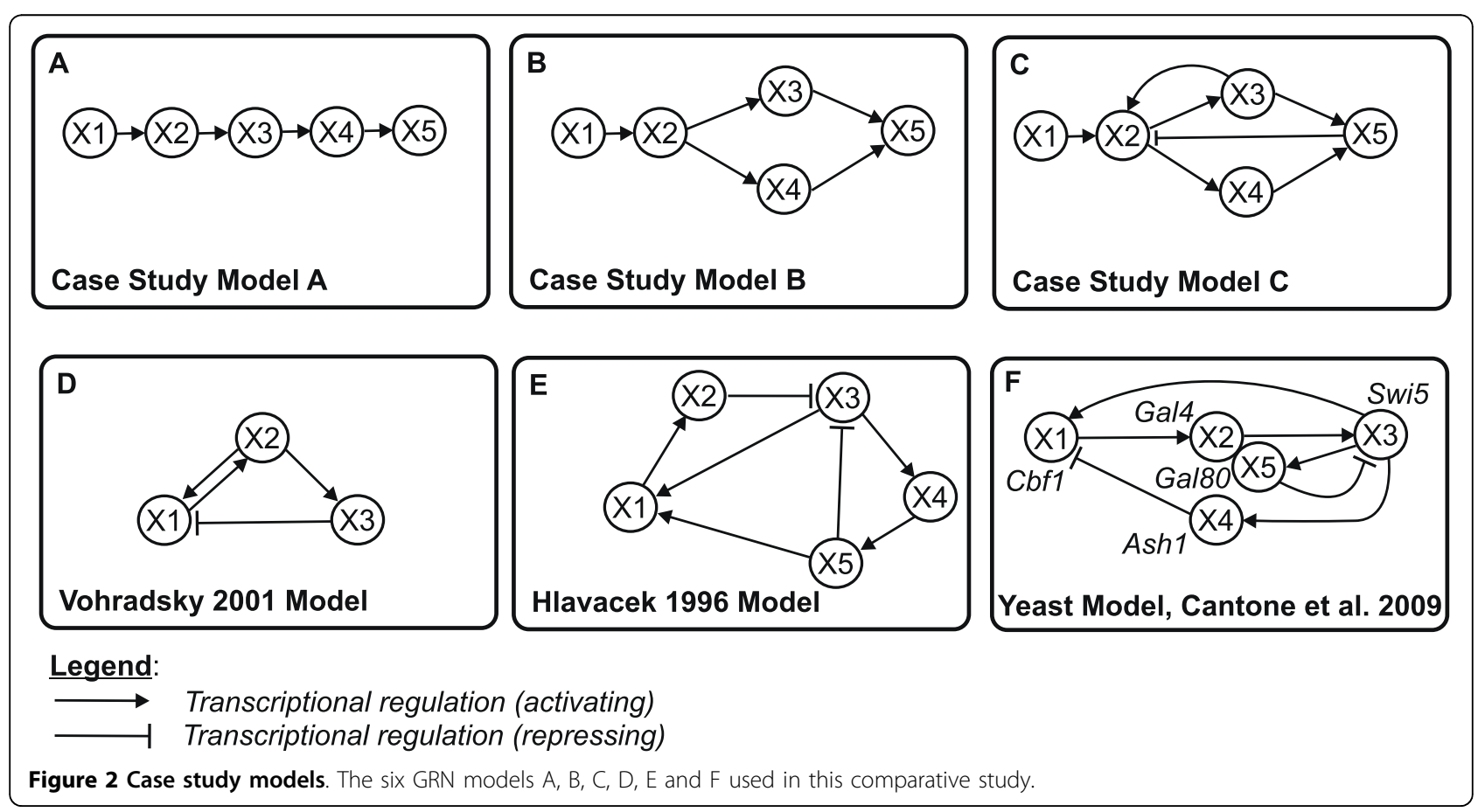


- Case A: This static GRN model describes a fourstep gene activation cascade starting from gene X1 up to gene X5. To generate harmonic dynamics with models of case A, we defined in all three methods (ANN, SS and GRLOT) constant signal propagation in the cascades by using identical parameter values within the parameter sets.

- Case B: With this model, we describe again a gene activation cascade, however, this case incorporates signal branching (multiple output) and co-regulatory relationships (multiple input) into the GRN. Here, gene $\mathrm{X} 2$ activates gene $\mathrm{X} 3$ and $\mathrm{X} 4$, both of which stimulate (activate) the expression of gene X5. We defined asymmetric signal branching such that the regulatory impact between $\mathrm{X} 2$ and $\mathrm{X} 3$ is different from the regulatory impact between $\mathrm{X} 2$ and $\mathrm{X} 4$. Furthermore, the co-regulated gene X5 is more sensitive to signals originating from $\mathrm{X} 4$ than to those of $\mathrm{X} 3$.

- Case C: The gene network described in the static GRN model $C$ extends the GRN described in scenario $\mathrm{B}$ by incorporating multi-component loops, a positive feedback loop between gene $\mathrm{X} 2$ and gene $\mathrm{X} 3$, and a negative feedback loop between the genes X2 and X5. The models describing case $\mathrm{C}$ simply extend the parameter sets of case $B$ with parameters defining stimulation between X3 and $\mathrm{X} 2$ and inhibition between $\mathrm{X} 2$ and X5. Thus, all parameter sets of case $C$ contain two closed-loop relationships, one positive feedback loop between $\mathrm{X} 2$ and $\mathrm{X} 3$, and one negative feedback loop between $\mathrm{X} 2$ and $\mathrm{X} 5$.

The common characteristics shared by the different regulatory parameter matrices (arising from $N$ coupled differential equations that represent a dynamic GRN model) are summarized as follows:

1. Case A: Uniform degradation rate (0.3), and constant signal propagation.

2. Case B: Uniform degradation rate (0.3), asymmetric signal branching and asymmetric coregulation.

3. Case C: Uniform degradation rate (0.3), negative and positive feedback loops.

We use these characteristics as evaluation criteria in our qualitative analysis of the accuracy of each reverseengineered model (Section 4.5.3). All model parameters are provided in Table 2 . The increasing complexity of the scenarios with their different regulatory mechanisms allowed us to gradually analyze and compare the individual modeling systems.

\subsubsection{Case studies $D$ and $E$}

The following two GRN scenarios (D and E) were taken from the literature $[15,21]$. These are depicted in Figure 2:

- Case D (V): The 3-gene static GRN model describing case $\mathrm{D}$ was used elsewhere to discuss the influence of the external input parameter $\vartheta_{i}$ on the dynamics of an ANN GRN model [15]. In this example $\vartheta_{i}$ is interpreted as delaying the reaction kinetics. Figure 3 shows two dynamic gene expression behaviors obtained by small variations in this parameter. This 3-gene GRN model comprises a positive and negative feedback regulation where products of gene $\mathrm{X} 1$ activate the expression of gene $\mathrm{X} 2$ and products of gene X2 stimulate the expression of gene X3 and $\mathrm{X} 1$. Gene X3 inhibits the expression of gene X1.

- Case E (HS): The fifth GRN system is the 5-gene static GRN model used by Hlavacek and Savageau [21]. This model is frequently used to evaluate reverse-engineering algorithms in combination with the SS method $[27,33,37]$. The dynamic behavior of this model is strongly influenced by singularities allowed by the SS method. In this scenario, singularities can be caused by the inhibitory dependency between gene $\mathrm{X} 5$ and $\mathrm{X} 3$ when the concentration level of X5 reaches zero.

We use the two cases (D and E), which were specifically generated for the ANN and SS methods, respectively, to provide additional evidence for the idiosyncratic behavior of the investigated modeling methods.

\subsubsection{Case study $F$}

This biological GRN was included in this study to ensure that the results we obtain from our artificial networks can be transferred to real biological systems. It was constructed synthetically in yeast by Cantone et al. [19] to facilitate an in vivo assessment of various reverse-engineering and gene network modelling approaches, including approaches based on ordinary differential equations.

Cantone et al. present expression profiles of the network genes after a shift from glucose- to galactose-raffinose-containing medium: this is called the switch-on time series; and after a shift from galactose-raffinose to glucose-containing medium: the switch-off time series. In this study we used the first 100 minutes of these two data sets, excluding the first 10 minute interval during which the washing steps and subsequent medium shift are performed. After 100 minutes the biological system is perturbed, and Cantone et al use time-delay terms to model this perturbation. We have not explored timedelay terms in our mathematical systems and so we do not model this perturbation. 
Table 2 Method parameters for case studies

\begin{tabular}{|c|c|c|c|c|c|c|c|c|c|c|c|c|c|c|c|}
\hline & \multicolumn{7}{|c|}{ The SS method } & \multicolumn{8}{|c|}{ The ANN method } \\
\hline & $\alpha$ & $\beta$ & $\mathrm{X} 1$ & $\mathrm{X} 2$ & $\mathrm{X3}$ & $\mathrm{X} 4$ & $\mathrm{X} 5$ & $v_{i}$ & $\theta$ & $k$ & $\mathrm{X} 1$ & $\mathrm{X} 2$ & $\mathrm{X} 3$ & $\mathrm{X} 4$ & $\mathrm{X5}$ \\
\hline \multicolumn{16}{|l|}{ Case A } \\
\hline $\mathrm{X} 1$ & 0.0 & 0.3 & - & - & - & - & - & 1.0 & 4.0 & 0.3 & - & - & - & - & - \\
\hline $\mathrm{X} 2$ & 1.0 & 0.3 & 2.0 & - & - & - & - & 1.0 & 4.0 & 0.3 & 5.0 & - & - & - & - \\
\hline $\mathrm{X} 3$ & 1.0 & 0.3 & - & 2.0 & - & - & - & 1.0 & 4.0 & 0.3 & - & 5.0 & - & - & - \\
\hline$X 4$ & 1.0 & 0.3 & - & - & 2.0 & - & - & 1.0 & 4.0 & 0.3 & - & - & 5.0 & - & - \\
\hline$\times 5$ & 1.0 & 0.3 & - & - & - & 2.0 & - & 1.0 & 4.0 & 0.3 & - & - & - & 5.0 & - \\
\hline \multicolumn{16}{|l|}{ Case B } \\
\hline $\mathrm{X} 1$ & 0.0 & 0.3 & - & - & - & - & - & 1.0 & 4.5 & 0.3 & - & - & - & - & - \\
\hline$\times 2$ & 1.0 & 0.3 & 1.5 & - & - & - & - & 1.0 & 4.5 & 0.3 & 7.0 & - & - & - & - \\
\hline$x 3$ & 1.0 & 0.3 & - & 2 & - & - & - & 1.0 & 4.5 & 0.3 & - & 6.0 & - & - & - \\
\hline$X 4$ & 1.0 & 0.3 & - & 3 & - & - & - & 1.0 & 4.5 & 0.3 & - & 4.0 & - & - & - \\
\hline$\times 5$ & 1.0 & 0.3 & - & - & 2 & 3 & - & 1.0 & 4.5 & 0.3 & - & - & 4.0 & 6.0 & - \\
\hline \multicolumn{16}{|l|}{ Case C } \\
\hline $\mathrm{X} 1$ & 0.0 & 0.3 & - & - & - & - & - & 1.0 & 4.5 & 0.3 & - & - & - & - & - \\
\hline$\times 2$ & 1.0 & 0.3 & 1.5 & - & 0.6 & - & -0.3 & 1.0 & 4.5 & 0.3 & 7.0 & - & 7.0 & - & -8.0 \\
\hline $\mathrm{X} 3$ & 1.0 & 0.3 & - & 2 & - & - & - & 1.0 & 4.5 & 0.3 & - & 6.0 & - & - & - \\
\hline$\times 4$ & 1.0 & 0.3 & - & 3 & - & - & - & 1.0 & 4.5 & 0.3 & - & 4.0 & - & - & - \\
\hline$\times 5$ & 1.0 & 0.3 & - & - & 2 & 3 & - & 1.0 & 4.5 & 0.3 & - & - & 4.0 & 6.0 & - \\
\hline \multicolumn{16}{|c|}{ The GRLOT method } \\
\hline & V & k & $\mathrm{X} 1 \mathrm{n}$ & $\mathrm{X} 1 \mathrm{ki}$ & $\times 2 n$ & X2 ki & $\times 3 n$ & X3 ki & $X 4 n$ & X4 ki & $\times 5 n$ & X5 ki & & & \\
\hline \multicolumn{16}{|l|}{ Case A } \\
\hline $\mathrm{X} 1$ & 0.0 & 0.3 & - & - & - & - & - & - & - & - & - & - & & & \\
\hline$\times 2$ & 1.0 & 0.3 & 1.5 & 1.1 & - & - & - & - & - & - & - & - & & & \\
\hline $\mathrm{x} 3$ & 1.0 & 0.3 & - & - & 1.5 & 1.1 & - & - & - & - & - & - & & & \\
\hline$\times 4$ & 1.0 & 0.3 & - & - & - & - & 1.5 & 1.1 & - & - & - & - & & & \\
\hline$x 5$ & 1.0 & 0.3 & - & - & - & - & - & - & 1.5 & 1.1 & & & & & \\
\hline \multicolumn{16}{|l|}{ Case B } \\
\hline $\mathrm{X} 1$ & 0.0 & 0.3 & - & - & - & - & - & - & - & - & - & - & & & \\
\hline$\times 2$ & 1.0 & 0.3 & 1.5 & 0.8 & - & - & - & - & - & - & - & - & & & \\
\hline$x 3$ & 1.0 & 0.3 & - & - & 2.5 & 0.5 & & - & - & & & & & & \\
\hline$X 4$ & 1.0 & 0.3 & - & - & 1.5 & 0.8 & & - & & & & & & & \\
\hline$\times 5$ & 1.0 & 0.3 & - & - & - & - & 1.5 & 0.8 & 2.5 & 0.5 & & & & & \\
\hline \multicolumn{16}{|l|}{ Case C } \\
\hline $\mathrm{X} 1$ & 0.0 & 0.3 & - & - & - & - & - & - & - & - & - & - & & & \\
\hline$x 2$ & 1.0 & 0.3 & 1.5 & 0.8 & - & - & 3.5 & 0.1 & - & - & -1.5 & 2.0 & & & \\
\hline X3 & 1.0 & 0.3 & - & - & 2.5 & 0.5 & - & - & - & - & - & - & & & \\
\hline$X 4$ & 1.0 & 0.3 & - & - & 1.5 & 0.8 & - & - & - & - & - & - & & & \\
\hline X5 & 1.0 & 0.3 & - & - & - & - & 1.5 & 0.8 & 2.5 & 0.5 & - & - & & & \\
\hline
\end{tabular}

Parameters for each of the SS, ANN and GRLOT methods as used in case study models A, B and C.

The network includes a variety of regulatory interactions, thus capturing the behavior of larger eukaryotic gene networks on a smaller scale (Figure 2). It was designed to be minimally affected by endogenous genes and to transcribe its genes in response to galactose.
While the yeast GRN appears relatively simple, it is actually quite articulated in its interconnections, which include regulator chains, single-input motifs, and multiple feedback loops, which are generated by the combination of transcriptional activators and repressors. 

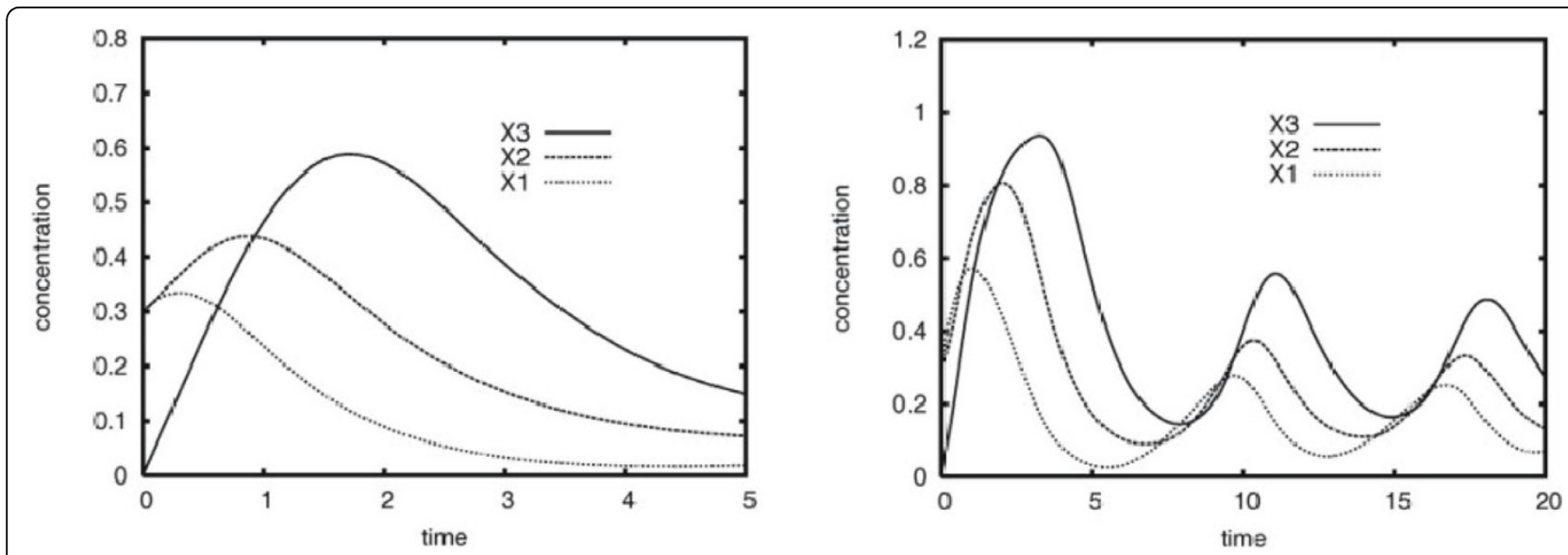

Figure 3 Dynamics of a 3-gene model. Various dynamics of the 3-gene model taken from Vohradsky (2001) [15], with $\bigotimes_{1}=3.0$ (left) and $\nabla_{1}=$ 1.0 (right). Small variations in the ANN parameter $\bigotimes_{i}$ cause different dynamics with constant topology parameters.

\section{Results and Discussion}

As we performed many different experiments, we first clarify how the different stages of our study contribute to our final set of results before discussing them in detail.

\subsection{Results}

Figure 1 describes the study design we adopted to compare the three modeling methods. Here we briefly describe the inputs and outputs of each step along with the relevant result tables and figures.

\section{Step 1: Construction of reference models}

Input: Three 5-gene static GRN models (models A, B, C), comprising regulatory cascades, single input modules, multiple input modules and multi-component loops $[35,36]$. Three models taken from the literature: a 3-gene static GRN model (D) [15], a 5-gene static GRN model (E) [21], and a 5-gene biological GRN (model F) synthetically generated in yeast [19].

Output: 13 dynamic GRN models:

- Nine 5-gene dynamic GRN models based on the static models A, B and C. For each static model, three dynamic models were constructed using the modeling methods ANN, SS and GRLOT, respectively.

- One 3-gene dynamic GRN model based on static model D, using the ANN method. The model parameters were taken directly from the literature [15].

- One 5-gene dynamic GRN model based on static model E, using the SS method. The model parameters were taken directly from the literature [21].

- Two 5-gene dynamic GRN models based on the switch-on and switch-off time series data sets presented in the literature [19].
Step 2: Generation of the artificial GRN expression data

Input: The 11 artificial GRN models from Step 1 (based on models A-E).

Output: 20 dynamic gene expression data sets:

- 11 sparse data sets based on simulating each GRN model with a single set of initial conditions.

- 9 detailed data sets from the first nine models of Step 1 (corresponding to the static GRN models A, $B$ and $C$, respectively). Each data set consists of 5 time series each created with different initial conditions.

\section{Step 3: Reverse-engineering of dynamic GRN models from}

\section{generated data}

Input: The output from Step 2, 20 dynamic gene expression data sets, plus the output from Step 1 for model F only, the switch-on and switch-off data sets: giving a total of 22 data sets.

Output: 66 dynamic GRN models. Each of the 22 data sets is reverse-engineered using the ANN, SS and GRLOT method, respectively.

\section{Step 4: Verification of reverse-engineered GRN models}

Input: 66 dynamic GRN models from Step 3.

Output: Calculation of training predictive power, $P_{\text {ver }}$, the inferential power, $P_{i n f}$, and qualitative and visual comparison of GRN features:

- Execution of 60 dynamic GRN for models A-E from Step 3 to determine $60 P_{v e r}$ values, each reflecting how well the model is able to predict (fit) the data from which it was generated.

- Calculation of the inferential power, $P_{\text {inf }}$, for 20 of the 60 models by comparing the reverse-engineered model parameters to the model parameters of the reference models from Step 1. This is possible only 
for model pairs, from Step 1 and Step 3, that share the same modeling method.

- Qualitative analysis of GRN features.

- Visual analysis of the models generated from the switch-on and switch-off data sets for the biological network (model F) as described in Section 2.2.1.

Table 3 and Table 4 summarize the results in terms of training (or verification) predictive power, $P_{v e r}$, and inferential power, $P_{\text {inf }}$, determined for the reverse-engineered ANN, SS and GRLOT models, when reproducing the network dynamics. The results of the qualitative analysis are presented in Table 5 .

Step 5: Validation of reverse-engineered GRN models Input:

- 54 of the 60 GRN models from Step 3 corresponding to the models $(\mathrm{A}, \mathrm{B}$, and $\mathrm{C})$ derived from nine sparse and nine detailed data sets using ANN, SS and GRLOT, respectively. Plus 9 reference GRN models, constructed in Step 1, corresponding to three ANN, three SS, and three GRLOT models, respectively.

- The 6 remaining GRN models from Step 3, corresponding to three for each of model D and $\mathrm{E}$.

- The 3 switch-on models from Step 3 for model F.

\section{Output:}

- 54 $P_{\text {val }}$ values indicating how well the reverse-engineered dynamic GRN models function under new conditions (i.e., reproduce unseen data). The results of the validation experiments with the reverse-engineered dynamic models are summarized in Table 3 and Table 4, respectively.
Table 4 Reverse-engineering results with data fitting scores only

\begin{tabular}{|c|c|c|c|c|}
\hline Sparse data & & ANN data & SS data & GRLOT data \\
\hline \multirow[t]{3}{*}{ to ANN model } & $P_{\text {ver }}$ & 0.9925 & 0.9909 & 0.9926 \\
\hline & $P_{\text {val }}$ & 0.9939 & 0.9907 & 0.9900 \\
\hline & $\Delta P_{\text {fit }}$ & 0.0014 & -0.0002 & -0.0026 \\
\hline \multirow[t]{3}{*}{ to SS model } & $P_{\text {ver }}$ & 0.9825 & 0.9986 & 0.9955 \\
\hline & $P_{\text {val }}$ & 0.6769 & 0.8822 & 0.6847 \\
\hline & $\Delta P_{\text {fit }}$ & -0.3056 & -0.1164 & -0.3108 \\
\hline \multirow[t]{3}{*}{ to GRLOT model } & $P_{\text {ver }}$ & 0.9737 & 0.9672 & 0.9975 \\
\hline & $P_{\text {val }}$ & 0.9712 & 0.9333 & 0.9970 \\
\hline & $\Delta P_{\text {fit }}$ & -0.0025 & -0.0339 & -0.0005 \\
\hline \multicolumn{5}{|l|}{ Detailed data } \\
\hline \multirow[t]{3}{*}{ to ANN model } & $P_{\text {ver }}$ & 0.9999 & 0.9846 & 0.9975 \\
\hline & $P_{\text {val }}$ & 0.9998 & 0.9789 & 0.9967 \\
\hline & $\Delta P_{\text {fit }}$ & -0.0001 & -0.0057 & -0.0008 \\
\hline \multirow[t]{3}{*}{ to SS model } & $P_{\text {ver }}$ & 0.9916 & 0.9999 & 0.9981 \\
\hline & $P_{\text {val }}$ & 0.8294 & 0.9917 & 0.8321 \\
\hline & $\Delta P_{f i t}$ & -0.1622 & -0.0082 & -0.1660 \\
\hline \multirow[t]{3}{*}{ to GRLOT model } & $P_{\text {ver }}$ & 0.9936 & 0.9934 & 0.9986 \\
\hline & $P_{\text {val }}$ & 0.9935 & 0.9879 & 0.9982 \\
\hline & $\Delta P_{\text {fit }}$ & 0.0001 & -0.0055 & -0.0004 \\
\hline
\end{tabular}

Data (created by ANN, SS, GRLOT methods) predicted by the different methods (for cases A, B, C) and subsequently used for making in silico predictions; for sparse and detailed data sets.

- By reproducing data sets based on the 5-gene Hlavacek model (model E) described with the SS method, we analyzed if the ANN and GRLOT methods can be used for reverse-engineering these dynamics without supporting regulation of the degradation process. Accordingly, we analyzed the Vohradsky model (model D) to see if small variations of the external

Table 3 Reverse-engineering verification and validation results for A, B and C

\begin{tabular}{|c|c|c|c|c|c|c|c|c|c|}
\hline & \multicolumn{3}{|c|}{ ANN data } & \multicolumn{3}{|c|}{ SS data } & \multicolumn{3}{|c|}{ GRLOT data } \\
\hline & \multicolumn{9}{|c|}{ Sparse data set } \\
\hline$P_{\text {inf }}$ & 0.9620 & 0.9701 & 0.8562 & 0.9308 & 0.9994 & 0.9947 & 0.9546 & 0.4820 & 0.6046 \\
\hline$P_{\text {ver }}$ & 0.9996 & 0.9797 & 0.9982 & 0.9998 & 0.9988 & 0.9973 & 0.9985 & 0.9941 & 0.9999 \\
\hline$P_{\text {val }}$ & 0.9972 & 0.9870 & 0.9976 & 0.9953 & 0.8635 & 0.7878 & 0.9982 & 0.9927 & 0.9999 \\
\hline \multirow[t]{2}{*}{$\Delta P_{\text {fit }}$} & -0.0024 & 0.0073 & -0.0016 & -0.0045 & -0.1353 & -0.2095 & -0.0003 & -0.0014 & 0.0000 \\
\hline & \multicolumn{9}{|c|}{ Detailed data set } \\
\hline$P_{\text {inf }}$ & 0.9896 & 0.9992 & 1.0000 & 1.0000 & 0.9999 & 1.0000 & 0.6704 & 0.7070 & 0.6432 \\
\hline$P_{\text {ver }}$ & 0.9998 & 0.9999 & 1.0000 & 1.0000 & 1.0000 & 1.0000 & 0.9983 & 0.9978 & 0.9987 \\
\hline$P_{\text {val }}$ & 0.9997 & 0.9999 & 1.0000 & 0.9978 & 0.9882 & 0.9891 & 0.9978 & 0.9972 & 0.9996 \\
\hline$\Delta P_{\text {fit }}$ & -0.0001 & 0.0000 & 0.0000 & -0.0022 & 0.0118 & 0.0109 & -0.0005 & -0.0006 & 0.0009 \\
\hline
\end{tabular}


Table 5 Parameter matrix characteristics

\begin{tabular}{|c|c|c|c|c|c|c|c|c|c|}
\hline \multirow[b]{2}{*}{ Data generation method } & \multicolumn{3}{|c|}{ Case A } & \multicolumn{3}{|c|}{ Case B } & \multicolumn{3}{|c|}{ Case $\mathrm{C}$} \\
\hline & ANN & SS & GRLOT & ANN & SS & GRLOT & ANN & SS & GRLOT \\
\hline Uniform degradation rate & $x--$ & $x \times x$ & $x \times x$ & $x--$ & $x \times x$ & $x \times x$ & $x \times x$ & $x \times x$ & $x \times x$ \\
\hline Constant signal propagation & $x--$ & $-x x$ & $-x x$ & & & & & & \\
\hline Asymmetric signal branching & & & & $x \times x$ & $-x x$ & $x \times x$ & $x--$ & $-x x$ & $-x x$ \\
\hline Asymmetric co-regulation & & & & 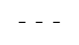 & $-x-$ & -- & - - - & $x x-$ & $--\cdot$ \\
\hline Negative feedback & & & & & & & $x \times x$ & $x \times x$ & $x \times x$ \\
\hline Positive feedback & & & & & & & $x x-$ & $x \times x$ & $x \times x$ \\
\hline Uniform degradation rate & $x--$ & $x \times x$ & $x \times x$ & $x \times x$ & $x \times x$ & $x \times x$ & $x \times x$ & $x \times x$ & $x \times x$ \\
\hline Constant signal propagation & $x--$ & $-x x$ & $-x x$ & & & & & & \\
\hline Asymmetric signal branching & & & & $x \times x$ & $-x x$ & $x \times x$ & $x--$ & $x x-$ & $x \times x$ \\
\hline Asymmetric co-regulation & & & & $x-x$ & $x \times x$ & $x-x$ & $x--$ & $x x-$ & $x \times x$ \\
\hline Negative feedback & & & & & & & $x \times x$ & $x \times x$ & $x \times x$ \\
\hline Positive feedback & & & & & & & $x \times x$ & $x \times x$ & $x \times x$ \\
\hline
\end{tabular}

Characteristics found in the parameter matrices of the reverse-engineered network models: Characteristics corresponding to the sparse data sets are depicted in the top rows of the table, those corresponding to the detailed data sets in the bottom rows. The symbol " $\mathrm{x}$ " shows that the feature was discovered by the method, "-" its was not discovered, and spaces indicate that the feature was not part of the case study. The reverse-engineering methods applied to each data set are in the order ANN, SS, GRLOT.

input parameter $\vartheta_{i}$, which lead to large effects in ANN models, could be reverse-engineered into models based on the SS and GRLOT method. These results are summarized in Figure 4.

- Table 6 describing the results of perturbation experiments performed on the 3 switch-on data sets of model F.

\subsection{Discussion}

In order to ensure that our results based on the artificial data sets are fully applicable to biological systems, we first discuss the results from reverse-engineering the biological data (model F). We then discuss scenarios where the same methods were used for both reverseengineering and data set creation: this allows us to check if the methods are able to accurately discover the

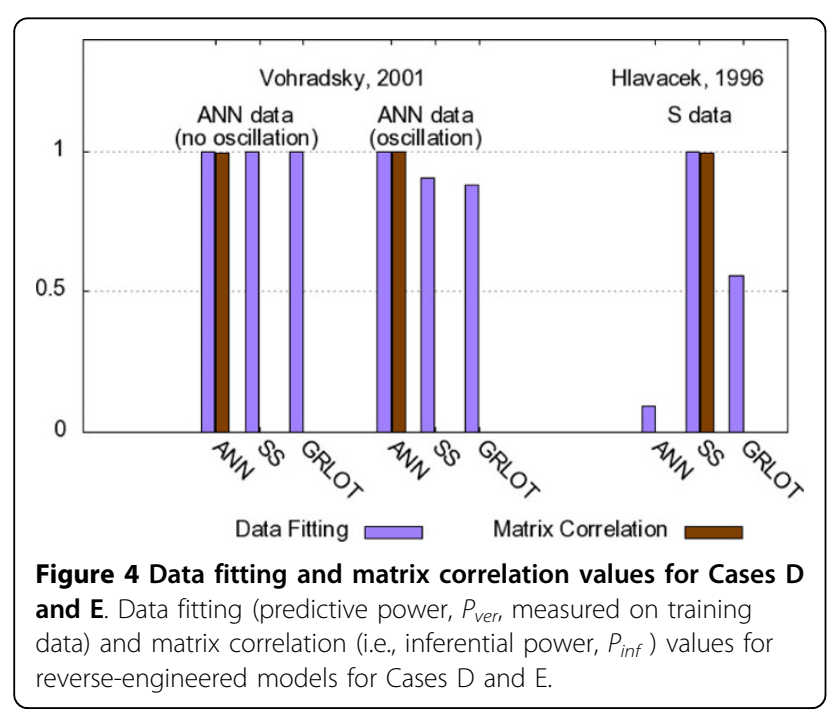

original parameter matrices used to generate the data and to see if the discovery of the parameter matrices is a necessary condition to generate accurate and realistic dynamics. Next we highlight idiosyncrasies between the methods by discussing the results when different methods were used for reverse-engineering and data set creation, and before concluding our discussion by considering specific features of the network models, we describe the results of our qualitative comparisons.

\subsubsection{Reverse-engineering biological data}

The ten graphs in Figure 5 show the gene expression data of the five genes in the model F, CBF1, GAL4, SWI5, GAL80, ASH1 [19], along with the dynamics predicted by the three methods. The ANN and GRLOT methods tend to provide a good match to the experimental data, with the ANN method giving the best results in this test. The SS is clearly worse than the other two methods due to the sensitivity of its many multiplied power terms. We have found that many of the terms automatically generated by the evolutionary algorithm for the SS method do not give any solution (i. e the integrator fails), and so the method therefore tends to converge on local minima where many, if not all, of the power terms are set to zero, thus resulting in simple linear graphs. However, sometimes a linear solution may be a reasonably accurate approximation to genes that are in a steady-state of expression, and so linear solutions may not have a significantly detrimental effect on the overall model output.

Table 6 summarizes the results for the perturbation experiments performed on the reverse-engineered models. The ANN and GRLOT are again in more accord with each other than they are with the SS method, and the SS method fails more frequently. Perturbing 
Table 6 Perturbation data for the models generated for the Cantone model F

\begin{tabular}{|c|c|c|c|c|c|c|c|}
\hline & & \multicolumn{3}{|c|}{ Comp. to unperturbed network } & \multicolumn{3}{|c|}{ Comp. to perturbed network } \\
\hline \multicolumn{2}{|c|}{ Perturbation } & ANN & GRLOT & SS & ANN to GRLOT & ANN to SS & GRLOT to SS \\
\hline \multirow[t]{4}{*}{ CBF1 } & 0.0045 & 1 & 1 & 1 & 0.951 & 0.461 & 0.337 \\
\hline & 0.0135 & 0.656 & 0.556 & 0.556 & 0.949 & 0.431 & 0.345 \\
\hline & 0.054 & 0.059 & 0.0397 & 0.0397 & 0.795 & 0.613 & 0.808 \\
\hline & 0.216 & 0.0035 & 0.0022 & - & 0.0072 & - & - \\
\hline \multirow[t]{4}{*}{ GAL4 } & 0.0324 & 1 & 1 & 1 & 0.951 & 0.461 & 0.337 \\
\hline & 0.0972 & 0.940 & 0.549 & - & 0.542 & - & - \\
\hline & 0.3888 & 0.059 & 0.0395 & - & 0.0606 & - & - \\
\hline & 1.5552 & 0.00025 & 0.0023 & - & 0.0052 & - & - \\
\hline \multirow[t]{4}{*}{ SWI5 } & 0.0075 & 1 & 1 & 1 & 0.951 & 0.461 & 0.337 \\
\hline & 0.0225 & 0.992 & 0.994 & 0.893 & 0.952 & 0.310 & 0.222 \\
\hline & 0.09 & 0.800 & 0.838 & 0.217 & 0.953 & 0.0672 & 0.0506 \\
\hline & 0.36 & 0.180 & 0.221 & - & 0.953 & - & - \\
\hline \multirow[t]{4}{*}{ GAL80 } & 0.0221 & 1 & 1 & 1 & 0.951 & 0.461 & 0.337 \\
\hline & 0.0663 & 0.541 & 0.498 & 0.371 & 0.930 & 0.555 & 0.545 \\
\hline & 0.2652 & 0.0208 & 0.0354 & 0.0192 & 0.915 & 0.581 & 0.545 \\
\hline & 1.0608 & $2.09 \mathrm{e}-05$ & 0.0021 & - & 0.0022 & - & - \\
\hline \multirow[t]{4}{*}{ ASH1 } & 0.012 & 1 & 1 & 1 & 0.951 & 0.461 & 0.337 \\
\hline & 0.036 & 0.939 & 0.935 & 0.780 & 0.951 & 0.471 & 0.342 \\
\hline & 0.144 & 0.338 & 0.328 & 0.212 & 0.950 & 0.476 & 0.344 \\
\hline & 0.576 & 0.0272 & 0.0263 & 0.037 & 0.945 & 0.471 & 0.338 \\
\hline
\end{tabular}

Perturbation data for the models generated for the Cantone model F [19], based on the switch-on network only. The left hand set of results compares the perturbed network to the unperturbed network: they give an indication of how the perturbation has changed the network dynamics. The right hand set of results compares the perturbed networks across the three methods. The closer the values are to 1.0, the more similar the dynamics. The perturbations involve increasing the initial gene values by $\times 3, \times 12$, and by $\times 48$.

different genes influences the network dynamics in different ways, some giving a large change to the overall network dynamics, and some leaving them relatively unchanged. This is shown in the table by the comparing the perturbed network dynamics to the unperturbed state. When comparing the predicted network dynamics for the different methods, the results indicate that good agreement is possible between the ANN and GRLOT methods, especially for the smallest perturbations, but also sometimes even for the largest perturbations we explored: however, this is not always the case as there was little agreement between the methods when the GAL4 gene was perturbed. We believe that these results show that the mathematical methods are able to generate reasonable solutions for biological data sets. In subsequent sections we further elucidate characteristics of the methods using our more detailed artificial data sets.

\subsubsection{Within-method estimation of model parameters}

We examined the accuracy of model parameter estimation for the situation where the reference GRN model (constructed in Step 1) and the reverse-engineered GRN model are based on the same modeling method (ANN, SS or GRLOT). To assess how accurately the original parameter matrices were discovered, we used the inferential power measure, $P_{i n f}$, defined by Equation 13.
Table 3 depicts the results we obtained from estimating within-method model parameters of the reverse-engineered models. First, we observe that although the ANN and GRLOT methods achieved relatively low $P_{i n f}$ scores (compared to the SS method), they still performed relatively well in terms of predicting the training data measured by the $P_{\text {ver }}$ scores. The GRLOT method is particularly notable here: For case $C$ on the sparse data set, even though the $P_{\text {inf }}$ value is only 0.6046 , the $P_{v e r}$ value is 0.9999 , indicating very accurate replication of the model dynamics even with significant errors in the parameter matrices. Indeed, this model also makes highly accurate predictions when it is perturbed by increasing and decreasing inputs. This is in marked contrast to models based on the SS method, which performed relatively well at discovering the original parameters (high $P_{i n f}$ ), but made poor predictions (low $P_{\text {val }}$ ) when the model was perturbed. This indicates a lack of robustness of the SS method due to its extreme sensitivity. Compared to the SS method, models based on both the ANN and GRLOT methods are much more robust to variations in their model parameters and are thus able to make more accurate predictions (higher $P_{\text {val }}$ ) of model behavior.

The experiments with the detailed data sets confirmed these results. As expected, all methods perform better 

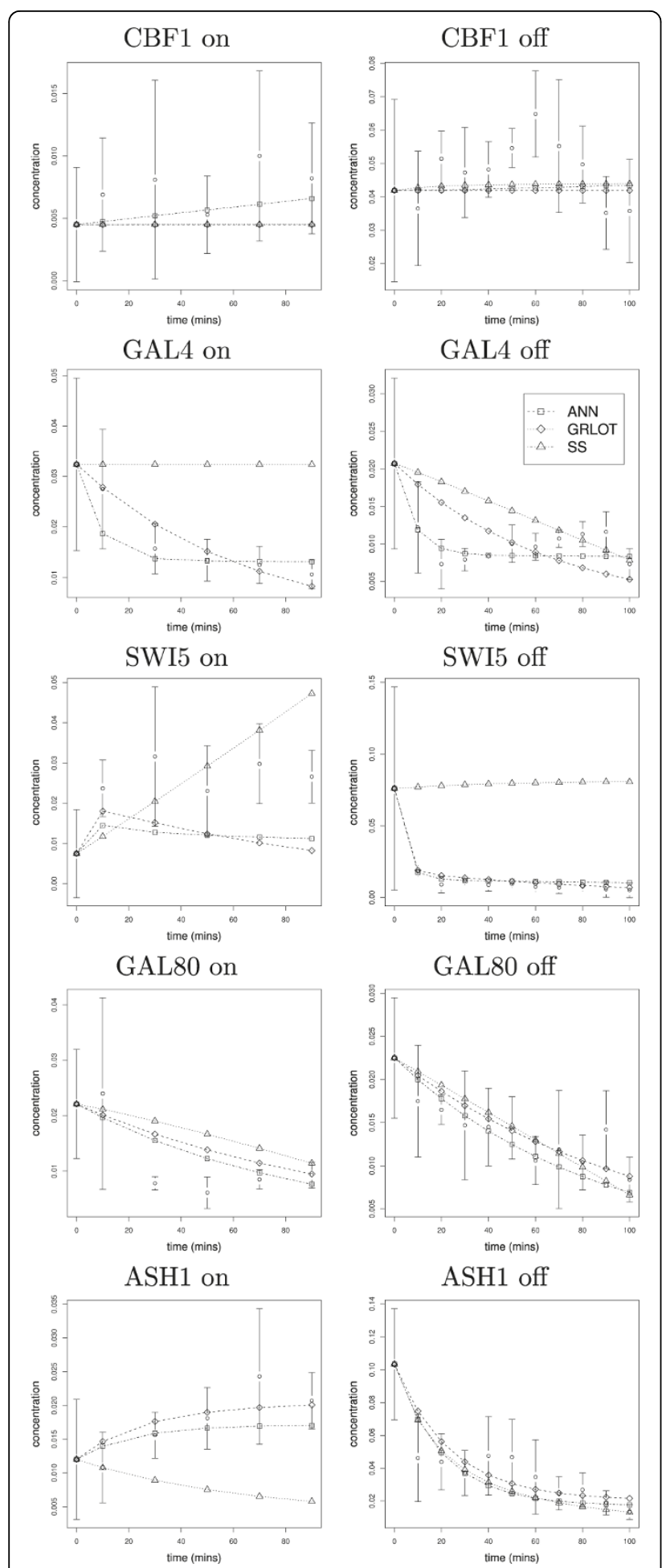

Figure 5 Switch-on and switch-off dynamics of GRN model F. Based on the synthetic network and corresponding data sets from Cantone et al. [19] we show the experimentally determined data with error bars for a switch-on and switch-off times-series, along with the modelled gene expression curves generated by each of the three methods. with the more detailed data set (providing more training data reflecting different initial conditions), and even the SS method improved the quality of its predictions, but it was still outperformed by the ANN and GRLOT methods.

\subsubsection{Reverse-engineering different method data}

Looking at the situation when the methods reconstructed (predicted) all data sets generated by all the methods, we relied on the $P_{v e r}$ measure as the methods were unable to reverse-engineer the original parameter matrices in all cases. The results of these experiments are summarized in Table 4.

As expected, the quality of the models is lower when the methods predicted the data created by one of the other methods. The SS method is conspicuous in its poor performance. In Table 3, the $P_{i n f}$ values for the SS method indicate that the parameter matrix was discovered with very high precision (the values are 0.9994 for sparse data and 0.9999 for detailed data). However, the $\Delta P_{f i t}$ values, which indicate the performance of the models when making predictions about network behavior under changes in input, are very poor: the values for cases $B$ and $C$ are 0.1358 and -0.2095 , respectively, for the sparse data and 0.0118 and 0.0109 , respectively, for the detailed data. Bearing in mind that a $\Delta P_{f i t}$ value of 0.1000 or above is of poor quality, with almost no visible relation to the original or expected dynamics, and that accurate models would require a $\Delta P_{f i t}$ value of about 0.0100 or less (see in Section 4.5.1), it then becomes clear that the ability of the SS method to make satisfactory predictions about network behavior is much less than that of the ANN and GRLOT methods, even when highly sampled data of near-perfect accuracy is used (i. e., artificial data created by the SS method). This inaccuracy is due to the exponential terms in the SS method, which are highly sensitive to its parameters even when they are accurate to three or four decimal places. A decrease in model input corresponds to an increase in the degradation rate $k_{1}$ (or $\beta_{1}$ ) on gene $X_{1}$, as described in Section 4.4. Due to the nature of the dynamics, the $k_{1}$ increase is a smaller perturbation than the decrease, corresponding to the differences in these average values for $k_{1}=0.5000$ and $k_{1}=0.1000$, respectively. The individual data for input increase and decrease predictions show that the SS method actually performed as well as the other two models when the input was decreased, the problems arose when the input was increased. The results therefore indicate that the SS models may be reasonably robust to small perturbations, but are highly sensitive to larger perturbations and consequently liable to lose all predictive ability.

It is also worth noting that the SS method performed poorer in reverse-engineering well-performing GRN 
models based on data generated by the GRLOT and ANN methods than the GRLOT and ANN methods in reverse-engineering models based on the data created by the SS method. The ANN and GRLOT also performed slightly worse when reverse-engineering from SS data than when reverse-engineering from data created by the GRLOT or ANN method, respectively.

\subsubsection{Qualitative comparison of network features}

The results of the qualitative comparison between the methods, as described in Section 4.5.3, are summarized in Table 5. They show that the GRN models reverseengineered from the detailed data sets had a higher similarity to the original models, in terms of their underlying network features, than models based on the sparse data set. This result portrays the opposite of what is observed on the basis of $P_{v e r}$ values, where the reverse-engineered models score higher with the sparse data than the detailed data sets. This result is an indication of the problems that arise with discovering the underlying network features when the data availability is low.

In the following we consider different network features individually:

Uniform degradation rate With reference to sparse data, Table 5 (block of top rows), the results show that the ANN method was highly successful in discovering uniform degradation rates in each of the three cases and with each data set. However, the other two methods performed better in predicting data generated by the SS or GRLOT method than by the ANN method. Even with detailed data, the SS and GRLOT methods failed to discover the uniform degradation rate as simulated by the ANN method, especially with case A, although all methods discovered this feature when generated by the SS and GRLOT methods.

Constant signal propagation Only case A has the constant signal propagation feature. There is a clear difference between the methods: The ANN method was able to reproduce its own data, but not that of the SS or GRLOT method, whereas the SS and GRLOT could reproduce their own and each other's data, but not the data generated by the ANN method.

These findings can be explained by the different way in which the three methods process inputs. For example, constant signal propagation from one gene to another described with uniformly weighted input values (as realized by the ANN method) can certainly not be reproduced with constant signal propagation defined with uniformly exponentiated input values (SS method).

Asymmetry in signal branching and co-regulation Asymmetric signal branching and co-regulation are features in the case studies B and C. For sparse data, the methods were less successful at discovering asymmetric co-regulation than asymmetric signal branching, but for detailed data both features were modeled with similar success rates. As with the case of constant signal propagation, the different approaches to input processing of the three methods seem to explain this observation.

For sparse data, the asymmetric co-regulation feature was not discovered at all when reverse-engineering from data generated by the ANN and GRLOT method, respectively. In case $\mathrm{B}$, co-regulation involves the interplay between the two genes, $\mathrm{X} 3$ and $\mathrm{X} 4$, that control the expression of gene X5. By looking into the details of the models (data not shown here), the ANN and GRLOT models either described co-regulation with interchanged asymmetry or contain, incorrectly, an inhibitory dependency of gene X5. The sparse data set was not sufficient to ensure that this characteristic was reflected in the reverse-engineered models, but for detailed data the methods were more successful and in particular the ANN method could correctly reproduce co-regulation for all the data sets.

Positive and negative feedback loops These features are only found in the static GRN of case C. The methods have performed relatively well in reverse-engineering these features. Specifically, all models reverseengineered from one time-series (Table 5 , block of rows at the top) contain the positive and the negative feedback loops of network case $\mathrm{C}$, with the exception of one GRLOT model that was reverse-engineered from ANN data. This GRLOT model discovered an extra negative feedback loop instead of the positive feedback loop.

All models reverse-engineered from the detailed data rediscovered the feedback loops. Feedback loops play a key role in the model dynamics of network case $C$, and are therefore essentially contained in nearly all reverseengineered models.

\subsubsection{Considering the different case studies}

This section analyzes the different case studies in some detail.

Cases $A, B$, and $C$ Generally, the increasing complexity of the static GRN models from A to $C$ had no significant impact on the predictive power of the reverse-engineered models on the training data ( $P_{v e r}$ values). This, however, might be different in more complex reverseengineering settings where the network topologies are not known.

The Vohradsky case D: Oscillatory dynamics While models based on all three methods were able to obtain high $P_{v e r}$ values on reproduced non-oscillatory dynamics, the SS and GRLOT models reverse-engineered from the time series did not perform so well for oscillatory dynamics. The data sets used for these experiments are shown in the left and right panels of Figure 3.

The reason for the ANN method's ability to model oscillations lies in the external input parameters $\vartheta_{i}$ which can be used in ANN models to modulate the 
responsiveness of each gene to incoming signals individually. The ANN model used to generate the non-oscillatory dynamics used identical $\vartheta_{i}$ values whereas the underlying model of the oscillatory dynamics used different $\vartheta_{i}$ values to define fast and delayed signal responses. As shown in Figure 3, this led to oscillatory dynamics caused by slower responses within the cyclic negative feedback system defined in the Vohradsky scenario. These oscillations could not be reproduced by the reverse-engineered SS and GRLOT models (see Figure 6). Thus, the oscillatory dynamics is extremely biased towards the individual characteristics of the ANN method and are therefore difficult to model by dynamic GRN models based on the SS or GRLOT methods.

To better understand the data fitting verification method (based on the training data), it is useful to compare the time series shown in Figure 6 with the $P_{v e r}$ values given in Figure 4: the $P_{\text {ver }}$ values are still relatively high for the SS and GRLOT methods, despite their failure to reproduce the oscillatory dynamics seen in the left diagram of Figure 6.

The Hlavacek case E: singularities The same conclusions can be drawn from the results obtained with the Hlavacek case. The SS time series used here was also strongly influenced by unique characteristics of the SS method. First, the underlying network model of the test data set [38] involves the regulation of the degradation process of X3. More importantly, though, is the fact that singularities have a significant impact on the dynamics generated with this test model. As the $P_{v e r}$ values of Figure 4 show, this time series could therefore only be reproduced by the original SS method, whereas the ANN and GRLOT methods failed.

\section{Conclusions}

In this study we examined three systems of ordinary differential equations commonly applied in the modeling and reverse-engineering of GRNs. Our goal was to assess how the characteristics of the individual mathematical methods affect the quality of the reverse-engineered dynamic GRN models. Our results suggest that the methods are clearly not equivalent and interchangeable and that there are considerable differences in the way the methods process inputs. In particular, the SS method suffers from a relatively high degree of sensitivity to its parameters due to multiple input processing based on multiplied unrestricted exponentials. Thus, for the SS method, even when the original parameter matrix used to generate artificial test data sets was discovered with high accuracy the method sometimes failed to adequately reproduce the network dynamics.

Different methods seem to be suitable for modeling different types of dynamics or network features. For example, oscillatory dynamics may be modeled with the ANN method by using different values within its parameters. However, this may not possible with the SS and GRLOT models, which do not provide straightforward mechanisms for modeling oscillations.

Models reverse-engineered from under-sampled data, based on only a single experiment, are unlikely to discover the real parameter matrix, and should be regarded with considerable caution. Even when multiple, wellsampled time series data are available, it is does not seem wise to rely on models based on just a single modeling method, as it is likely to have some bias. The SS method, in particular, is prone to generating unrealistic dynamics, especially for relatively large input perturbations where our experiments showed its predicted output to be very inaccurate (see Section2.2.2). Even for very small gene networks, considerable amounts of highly time-resolved (many sampling points) and highly condition-resolved (based on multiple perturbations) data may be necessary to produce reliable dynamic GRN models. Furthermore, as the complexity (number of genes and regulatory interactions) increases, the computational complexity (compute power) required to reverse-engineer dynamic GRN models becomes nontrivial, and may require non-standard computing solutions such as clusters, supercomputers or other largescale computing solutions.

In the experiments we performed, SS-based dynamic GRN models generally yielded significantly less accurate
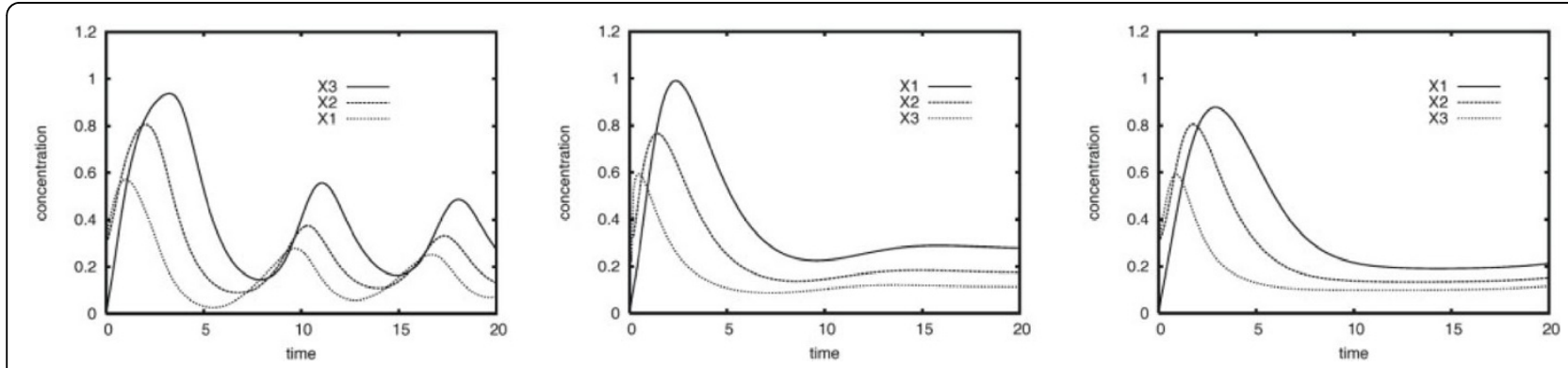

Figure 6 The oscillatory dynamics of three models. The oscillatory dynamics of the ANN model (left), cannot be reproduced in the SS and GRLOT models (middle) and (right). 
predictions than the ANN and GRLOT methods. While some features such as positive and negative feedback loops were correctly modeled in almost all situations we studied, it was often the case, especially when using the sparse data sets, that the methods failed to reverse-engineer features such as uniform degradation rates, constant signal propagation, asymmetry in signal branching and asymmetry in co-regulation. Even with the detailed data sets, these features could not be consistently reverse-engineered. Of the three methods, perhaps the ANN method deserves more attention due to its reliability and the relatively small number of parameters it requires. Indeed, although in this paper we have not presented results for the amount of computation required for each method, it is important to note that if the amount of computational power is limited, then the ANN method generally produces the most accurate and robust models for the least computation.

\section{Methods}

Before we describe the experimental design we used to compare the GRN modeling methods, we introduce some terminology.

\subsection{Terminology}

\section{Static GRN model}

The static model of a GRN represents the regulatory interaction structure or topology of a GRN. It describes whether or not there is a regulatory relationship between genes and what the nature of the interaction is, if it exists. The interaction nature is a binary property. It describes the influence of interacting genes, i.e., whether a gene has an activating (up-regulating, inducing) or inhibiting (down-regulating, repressing) influence on another gene. A static GRN model does not quantify gene expression amounts or regulatory influence. Static GRN models are easy to depict diagrammatically as a graph or network in which a node represents a gene and an edge a represents a regulatory interaction between two genes. The type and direction of an interaction is typically depicted symbolically: An arrow head represents activation, a bar represents inhibition. A diagrammatic depiction of the static GRN models used in our study is shown in Figure 2.

\section{Dynamic GRN model}

A dynamic GRN model refers to a representation that captures both the regulatory interaction structure (including the nature of the interactions) as well as quantitative rules that describe how gene expression amounts vary (in response to perturbations) over time.

\section{Dynamic gene expression data}

In general, dynamic gene expression data represent the time-variant expression levels (abundance of functional gene products: RNA, proteins) of the genes comprising a GRN. In our study we do not use dynamic gene expression data from real biological experiments. Instead, we generate dynamic gene expression data from dynamic GRN models with manually defined model parameters and (initial) expression levels.

\section{Reverse-engineering of GRNs}

Reverse-engineering of a GRN refers to the process of constructing (by automated means) a dynamic GRN model based on dynamic gene expression data. In particular, the reverse-engineering of a dynamic GRN model requires the reconstruction (from data) of the underlying regulatory interaction structure as well as the parameters governing the quantitative regulatory influence among genes. We simplified the reverse-engineering problem somewhat by avoiding the reverse-engineering of regulatory interactions that are not present in the static GRN models used as case studies (Figure 2).

\subsection{Model simulation: Generating the training data sets}

For the reference GRN models obtained from the static GRN models A, B and C, the initial concentration levels of the model variables $X_{i}$ are identical throughout all models and comprise five sets (called starting sets) leading to different dynamics (see Table 7). In the starting set 1 , for instance, the high initial concentration of X1 triggers the dynamics of the other network components, whereas the concentrations of starting set 5 are more evenly distributed.

The dynamics generated by the different modeling methods for the same GRN differ considerably. There are two main reasons for this: First, we chose model parameters producing different dynamics to provide a variety of expression profiles to facilitate the comparison of the three modeling methods. Here, the idea is to assess to what extent the reverse-engineered dynamic GRN models are able to reproduce the dynamics generated by the reference models. This relates to the model verification Step 4 in our study design. Second, the fact that the different reference models generate different dynamics is also explained by idiosyncratic characteristics of the modeling methods.

With the dynamic GRN reference models constructed from the static GRN models A, B and C the following dynamic gene expression data sets were generated (see Step 2 in our study design in Figure 1):

1. Sparse data sets, comprising the dynamic gene product concentrations of all genes but based on only a single set of initial conditions, namely starting set 1 (see Table 7).

2. Detailed data sets, based on five sets of initial conditions, namely based on starting set 1 to starting set 5 . 
Table 7 Initial model inputs

\begin{tabular}{llllll}
\hline & $\mathbf{X 1}$ & $\mathbf{X 2}$ & $\mathbf{X 3}$ & $\mathbf{X 4}$ & $\mathbf{X 5}$ \\
\hline Starting set 1 & 0.8 & 0.1 & 0.1 & 0.1 & 0.1 \\
\hline Starting set 2 & 0.8 & 0.1 & 0.1 & 0.3 & 0.5 \\
\hline Starting set 3 & 0.5 & 0.5 & 0.2 & 0.1 & 0.1 \\
\hline Starting set 4 & 0.4 & 0.8 & 0.2 & 0.2 & 0.2 \\
\hline Starting set 5 & 0.5 & 0.2 & 0.8 & 0.8 & 0.2
\end{tabular}

Initial mRNA concentration levels chosen for all models. We generate a sparse data set comprising one time-series based on starting set 1 and a detailed data set which comprises five time-series based on starting set 1 to starting set 5 .

3. All time series consist of 200 data points, each data point describing the gene product concentration $X_{i}\left(t_{k}\right)$ at time point $t_{k}$ for each gene $i=1, \ldots, n$.

The model parameters for the reference models of the Vohradsky model (case D), the Hlavacek model (case E), and the Cantone model (case F) were taken directly from the literature $[15,19,38]$.

\subsection{Reverse-engineering of dynamic GRN models from time-series data}

Many approaches to reverse-engineering of GRNs exist [12]. In our study reverse-engineering came down to a parameter estimation or optimization problem (Step 3 in our study design). We adopted an evolutionary algorithm approach to address this task [20]. Different reverse-engineered dynamic GRN models may be generated with different optimization techniques. However, we are confident that the parameter optimization technique we adopted was successful because, for the ANN and SS methods, we could demonstrate that the parameters of the reference models could be reverse-engineered with high accuracy (see the $P_{\text {inf }}$ values in Table 3 ). Furthermore, the validation results (predictive power on unseen data) for some of the dynamic GRN models we reverse-engineered with the evolutionary algorithm technique, show high levels of accuracy. Details of the optimization approach are discussed below.

\subsubsection{The parameter optimization problem}

The primary purpose of dynamic GRN models is to reveal regulatory mechanisms of biological gene regulation networks. Typically, in the case of highly abstracted GRN models as discussed here, dynamic (time-variant or time-series) gene expression data forms the input to the reverse-engineering process. When reverse-engineering dynamic GRN models from experimental time-series data, the problem is to identify model parameters for which the discrepancy between the experimental data and the data predicted by the model is minimal. This leads to an optimization problem in which the following function is to be minimized.

$$
f=\sqrt{\sum_{t=1}^{T} \sum_{k=1}^{N}\left(X_{k}(t)-\hat{X}_{k}(t)\right)^{2}}
$$

where

$X_{k}(t)$ denotes the experimentally observed gene product concentration level of gene $k$ at time point $t$.

$\hat{X}_{k}(t)$ denotes the gene product concentration level of gene $k$ at time point $t$ predicted by the dynamic GRN model.

$N$ denotes the number of genes in the network and $T$ the number of sampling points of observed data.

This approach is based on the assumption that the minimum of function $f$ corresponds to the set of parameters (i.e., parameter matrix) of the dynamic GRN model for which the model's outputs optimally fit the experimental data [28].

We performed optimization using evolutionary algorithms [20,39-41], which are stochastic search methods that mimic, on a highly abstracted level, biological evolution and sexual reproduction. Each evolutionary algorithm works with a population of points on the problem space where points are represented by individuals. Transformations on the individuals will tend to assemble individuals in more favorable areas of the search space, i.e., areas with high evolutionary fitness. When all or most individuals are located within the same area then a local (and possibly global) optimum of the search space is found.

Our approach was realized as follows. Individuals are represented by real-valued vectors, and each value can evolve within a defined variable-dependent interval. Here, the different genetic operators acting on those vectors ensure that the values remain with the specified intervals. First, we generated a random initial set or population of individuals. In this case, individuals are generated by setting random values to the unknown model parameters of the differential equations representing the dynamic GRN models (with the three modeling formalisms ANN, SS and GRLOT). A simulation was then performed for each individual and this was used to evaluate the fitness by comparing how well the simulated model output matches the time-series expression data (see Equation 5). The individuals were then ranked according to their fitness and in the next step we selected individuals for genetic operations, including cloning, recombination and mutation. These processes of selection, recombination and evaluation were repeated until a designated termination criterion was met, such as the attainment of an acceptable fitness level, or if a certain number of generations were evaluated. 


\subsubsection{The two-phase reverse-engineering strategy}

In this study, we applied a two-phase strategy to reverse-engineer dynamic GRN models from time-series gene expression data created in Step 2 from the reference models. We refer to the first phase as bottom-up phase and the second as top-down phase. We also employed the notions of a network model, denoted by $\mathbf{m}$, and a node model, denoted by $m_{k}$, to structure reverse-engineering problem [42].

Figure 7 illustrates the concepts of the node and network models. The node model describes the regulatory dependencies of a single gene, whereas the network models contain the dependencies of all genes of a network. Both types of model can be simulated: A network model requires only the initial conditions of constituent elements (i.e., gene product concentrations). In contrast, the node model requires as input the the time-dependent levels of all the elements. Consequently, node models usually fit the experiment very accurately, since they can use the experimental data as input, yet they are unable to predict behavior of the system under new experimental conditions.

The number of parameters to estimate in all three modeling methods ANN, SS and GRLOT increases with $O\left(N^{2}\right)$, where $N$ is the number of genes in the GRN. This makes it very difficult to reverse-engineer large-scale GRN models. Recently, a variation on the "network" model of reverse-engineering mentioned here was further developed based on the ANN method by Vohradsky and colleagues $[43,44]$. Even the reverse-engineering of relatively small GRNs, as presented by this study, turns out to be challenging. The advantage of node models, however, is that they require only $\frac{1}{N}$ th of the parameters required for the network model consisting of $N$ genes. Thus, a considerable complexity reduction can be achieved by decomposing the parameter optimization problem of a GRN into the smaller problem of $N$ node models with complexity $O(N)$. This problem decomposition approach was proposed as a step-by-step strategy by Maki and colleagues [45] and successfully applied elsewhere $[27,33,46]$.

Formally, we define a network model, m, as a structure comprising $N$ node models, $m_{k}$ as follows:

$$
\mathbf{m}=\left(m_{1}, m_{2}, m_{3}, \ldots, m_{N}\right) \quad k=1, \ldots, N
$$

Such a network model, $\mathbf{m}$, is capable of predicting the dynamics of a GRN consisting of $N$ genes. Each constituent node model, $m_{k}$ of $\mathbf{m}$, approximates the concentration changes, $d \hat{X}_{k} / d t$, captured by the experimental data according to the following function:

$$
\frac{d \hat{X}_{k}}{d t}=f\left(\mathbf{X}(t), \mathbf{w}_{\mathbf{k}}\right)
$$

where

$\frac{d \hat{X}_{k}}{d t}$ denotes the approximated gene product concentration changes of gene $k$,

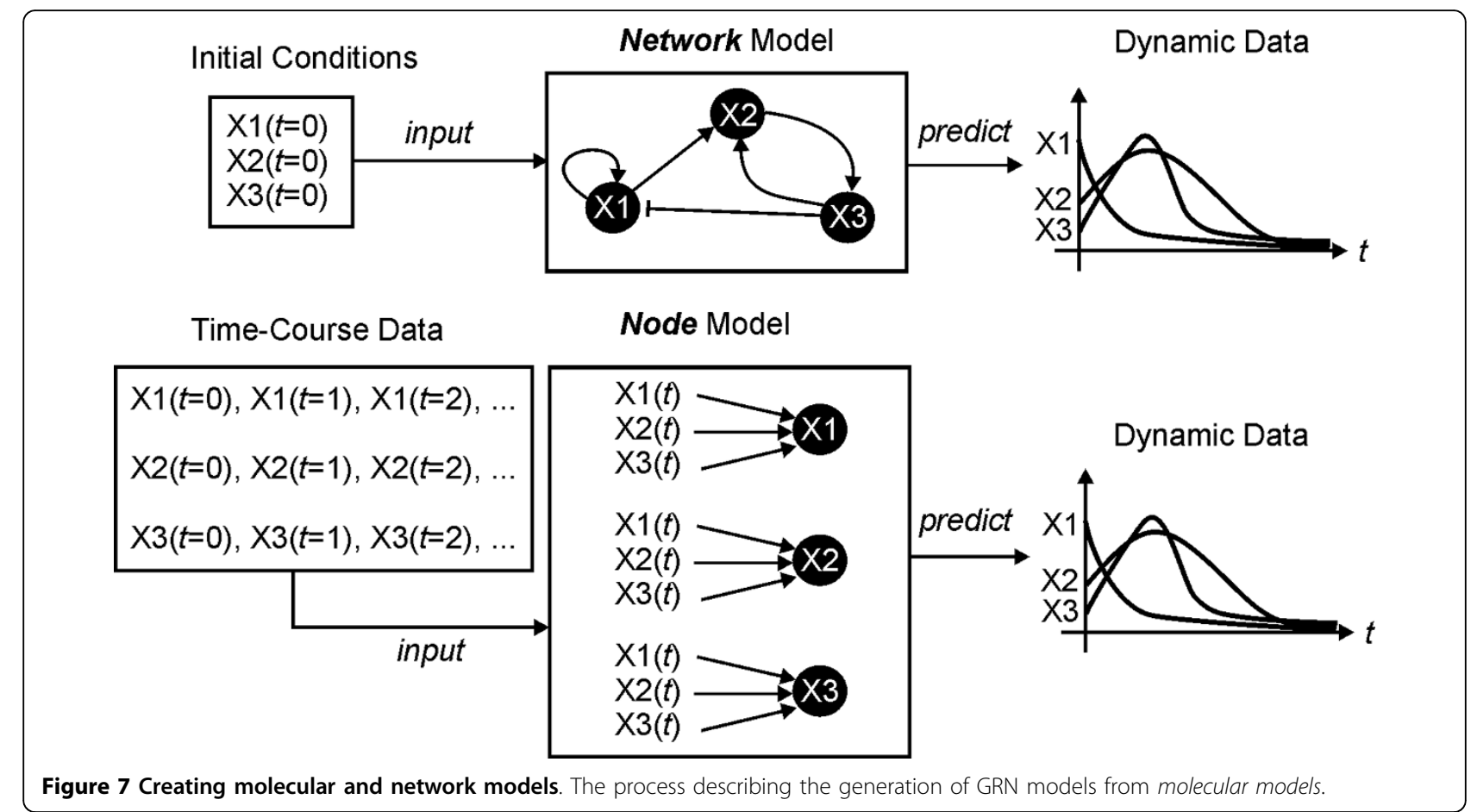


$\mathbf{X}(t)$ denotes the concentration levels of observed gene product concentrations at time $t$ as provided by the dynamic gene expression data,

$\mathbf{w}_{k}$ is a vector representing the parameters of the node model $m_{k}$.

To approximate the concentration dynamics of a gene within an entire network model, we define accordingly:

$$
\frac{d \hat{X}_{k}}{d t}=f\left(\mathbf{X}(0), \hat{\mathbf{X}}(t), \mathbf{w}_{k}\right)
$$

Here $\hat{\mathbf{X}}(\mathbf{t})$ describes the approximated concentration levels of the nodes (representing gene products) $k$ in the network and $\mathbf{X}(0)$ specifies the initial condition or state of the network model (as obtained from the time-series data).

In the bottom-up phase of our two-stage approach, we estimated the parameters $\boldsymbol{w}_{\boldsymbol{k}}$ of each individual node model, $m_{k}$, independent from the parameters of other node models. Subsequently, in the top-down stage, we reverse-engineered (optimized) the parameters, $\mathbf{W}$, of the entire network model, $\mathbf{m}$, by taking the results of the bottom-up approach as a starting point.

Bottom-up phase In the bottom-up phase the parameter estimation problem for one network model, $\mathbf{m}$, is divided into smaller parameter estimation problems for $N$ node models, $m_{k}$. The parameters, $\boldsymbol{w}_{\boldsymbol{k}}$, of the $N$ molecular models can be reverse-engineered step-by-step and independently from each other. This is facilitated by the extraction of the concentration levels, $X_{i}$, from all genes in the experimental data set, D. Hence, the objective function for reverse-engineering optimal node models from experimental data can be reduced to

$$
f_{k}=\sqrt{\sum_{t=1}^{p}\left(X_{k t}-\hat{X}_{k t}\right)^{2}}
$$

This function corresponds to the fitness of the $k$-th node model where $\hat{X}_{k t}$ is the calculated gene expression level of gene $k$ and $X_{k t}$ is the given expression level of gene $k$ at time $t$ from the data set $\mathbf{D}$.

With several repeated iterations of the bottom-up process, the intention was to find one or more optimal parameter sets $w_{k} i$ which produce small error values according to Equation 9. By combining the fittest of the $N$ identified node models form $m_{k}$, it is then possible to construct a self-contained network model, $\mathbf{m}$, which can simulate the dynamic behavior of the network independently from the gene expression matrix

D. Consequently, and in contrast to the node models $m_{k}$, a network model, $\mathbf{m}$, is capable of simulating or predicting scenarios (gene product concentrations) that are not captured by the experimental data.
Top-down phase In the top-down phase, we estimate parameter sets $\mathbf{W}=\left(w_{1}, w_{2}, \ldots, w_{\mathrm{N}}\right)$ of network models, m. Here, the concentration levels $\hat{\mathbf{X}}(t)$ and the parameters sets, $\mathbf{w}_{\mathrm{k}}$, of all node network models, $m_{k}$, in $\mathbf{m}$ are estimated together and dependent upon each other. So this case involves the interplay between the individual node networks in that the estimated concentration levels of each node network provide the potential input for other node models. In this case, the objective function for reverse-engineering optimal network models from experimental data is defined as

$$
f=\sqrt{\sum_{t=1}^{p} \sum_{k=1}^{N}\left(X_{k t}-\hat{X}_{k t}\right)^{2}}
$$

As already discussed, it is possible to construct a network model, $\mathbf{m}$, by merging of node models, $m_{k}$, found in the bottom-up phase. At this stage, however, the network model has not learned to work as a system that is aware of the interplay among the individual node models. Since the network model no longer derives its input from a static gene expression data matrix, $\mathbf{D}$, but instead is estimating the concentration levels for each network node, it can show dynamics totally different from the dynamics generated by the individual node models. The reason for this discrepancy lies in error propagation, where even small variations at the beginning of a simulation can cause dramatic fluctuations at later stages. For this reason, a network model constructed from node models is not the final result but rather constitutes a good starting position for the global parameter optimization.

\subsubsection{The parameter sets used}

To identify optimal parameter sets for the different gene expression models, we applied an evolutionary algorithm in which the genotype is directly based on real-valued vectors [41]. The genetic operators applied in our study are $B L X-\alpha$ crossover [47], with a crossover rate of 0.6 , and real number creep mutation, with a mutation rate of 0.1. The selection method used for selecting individuals for the generation changes is roulette-wheel selection with proportionality to the positions found in a rank-based fitness assignment operation. The number of individuals contained in one population was 500 and the number of generations was 300. Each parameter optimization for node models and for network models was repeated five times. This reverse-engineering strategy was able to precisely reproduce (often to more than 4 decimal places) the original parameter matrices for situations where the modeling method of reference model (Step 1) and reverse-engineered model (Step 3) were identical.

For consistency, we used the exact same approach (i. e., the same evolutionary algorithm parameters such as population size, number of generations, etc.) for all the reverse-engineering experiments. The only exception to 
this was the Cantone model $F$ that we worked on at a time when our usual infrastructure, described in Section 4.6, was not available. Instead we used a similar evolutionary algorithm on the Grid'5000 infrastructure [48].

The parameter space searched for the three methods was defined as follows:

- The ANN method: $w_{i j}$ from -15.0 to $15.0, v_{\mathrm{i}}$ from 0.0 to $3.0, \vartheta_{i}$ from 0.0 to 7.0 , and $k_{i}$ from 0.0 to 2.0.

- The SS method: $g_{i j}$ and $h_{i j}$ from -3.0 to 3.0, and $\alpha_{i}$ and $\beta_{i}$ from 0.0 to 15.0 .

- The GRLOT method: $I_{j}$, from -5.0 to $5.0, A_{k}$ from 0.0 to $7.0, K i_{j}$ and $K a_{k}$ from 0.0 to 40.0 , and $k_{i}$ from 0.0 to 2.0 .

\subsection{Model validation (Step 5)}

Finally, we performed a small number of experiments in which we first altered the initial conditions of the manually constructed reference models (Step 1), then simulated these models to generate dynamic gene expression data (serving as unseen validation data), and then investigated how the reverse-engineered dynamic GRN (Step 3) models behaved when identical perturbations were applied.

In all models, we varied the dynamics of gene product $\mathrm{X} 1$, by modifying the degradation rate $k_{1}$ in the GRLOT and ANN models, and the $\beta_{1}$ value in the SS models. This manipulation could be viewed as a variation of network input or network stimulus, since in all the case study models, X1 did not depend on the products of other genes.

Figure 8 shows the dynamics of the original gene product concentration $\mathrm{X} 1$, and an increased and decreased development of gene product concentration levels. The difference of the dynamics, as calculated by using Equation 12 , demonstrates the power (predictive power, $P_{v a l}$, on unseen data) of the reverse-engineered dynamic GRN models to reproduce the dynamic data generated by the manually created GRN reference models under different conditions.

For the Cantone model $\mathrm{F}$, the reverse-engineered models were used for perturbation experiments by perturbing the initial value of each gene in turn (whilst keeping the other initial values constant and equal to

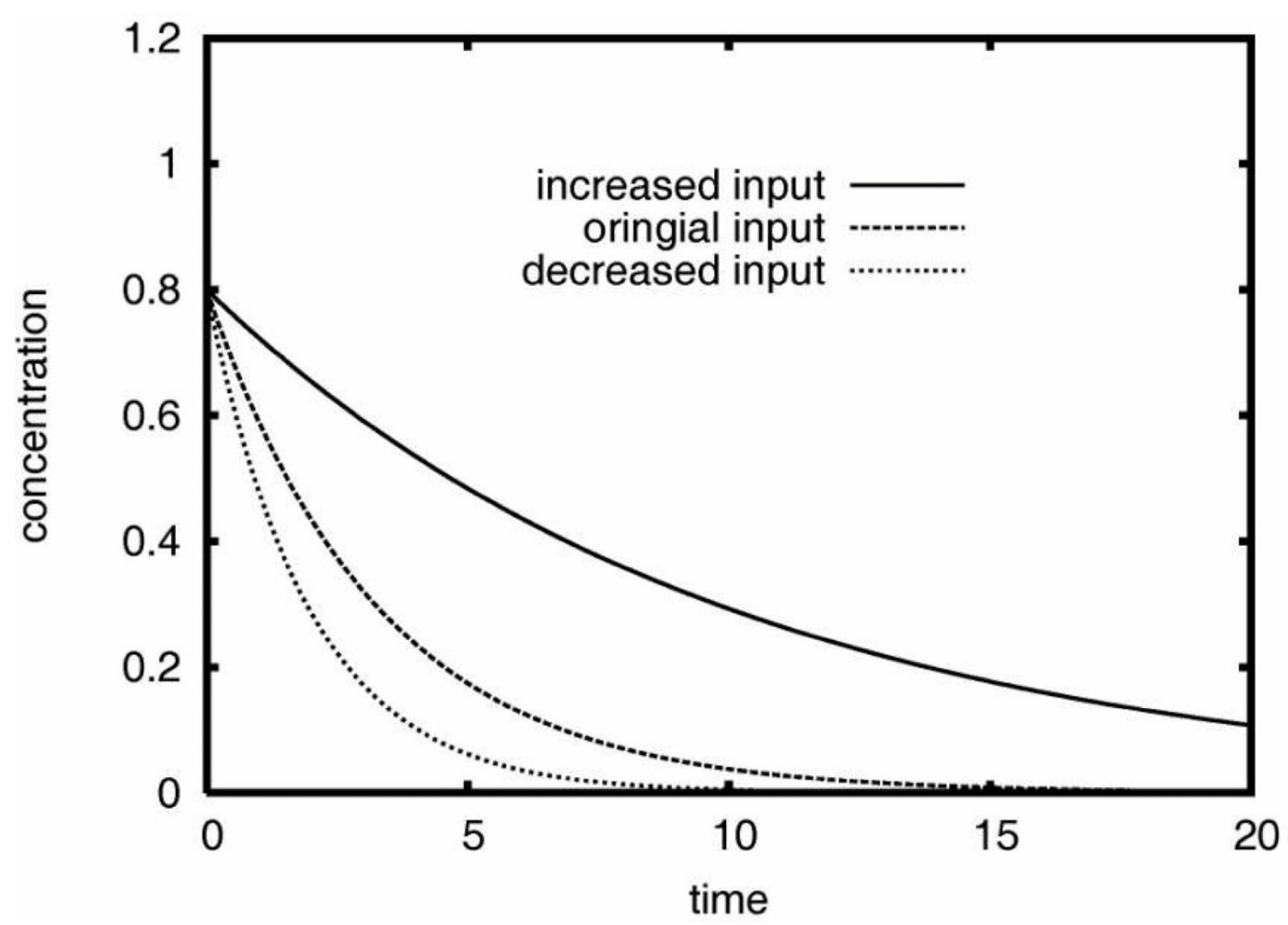

Figure 8 Dynamics with an input change. Dynamics of the original gene product concentration of X1 based on a degradation $k_{1}$ or $\beta_{1}$ of 0.3 . An increased transcript concentration is simulated by decreasing this value to 0.1 . Conversely, decreasing transcript concentration levels are simulated by increasing this value to 0.5 . 
their respective values for the initial time step in the two time-series data sets). The perturbed value was increased from its initial value by increasing it by $\times 3$, $\times 12$, and by $\times 48$. Equation 11 (which differs slightly from Equation 5) was used to calculate differences between simulated expression models:

$$
f=\sqrt{\sum_{t=1}^{p} \sum_{k=1}^{N}\left(\frac{X_{k t}-\hat{X}_{k t}}{X_{k t}}\right)^{2}}
$$

where, $\hat{X}_{k t}$ and $X_{k t}$ are the simulated mRNA levels at time $t$ of molecule $k . N$ is the number of genes in the network and $p$ is the number of sampling points of observed data.

\subsection{Measures to verify and validate the reverse- engineered GRN models}

Wessels and co-workers [28] defined measures for assessing the accuracy of reverse-engineered GRN models. Following their approach, we used the following measures (see also Step 4 and 5 of our study design):

- Predictive power, $P_{v e r}$, on training data. This measures how well a reverse-engineered dynamic GRN model reproduces the dynamic gene expression data from which it was generated. So the steps involved are: (a) Create training data from reference models (Step 2); (b) Reverse-engineer dynamic GRN models from training data (Step 3); (c) Execute or simulate reverse-engineered models (resulting in predicted dynamic gene expression data) and calculate $P_{v e r}$ by comparing the training data with the predicted data (Step 4).

- Predictive power, $P_{\text {val }}$, on unseen test data. This measures how well a reverse-engineered dynamic GRN model reproduces unseen dynamic gene expression data generated by the reference models under initial conditions different to those used to generate the training data. So the steps involved are: (a) Rerun the reference models (Step 1) under altered initial conditions to generate unseen test data; (b) Apply the same changed initial conditions to the dynamic GRN models generated by Step 3 and re-run the models under these conditions to predict dynamic gene expression data and calculate $P_{\text {val }}$ by comparing the unseen test data with the predicted data (Step 5).

- Predictive power, $P_{f i}$, for data fitting. We use $P_{f i t}$ when we refer to predictive power in a general way of fitting the data of a predicted data set with another (training or test) data set.
- Inferential power, $P_{\text {inf }}$, measures to what extent the parameters (representing GRN regulatory structure and quantitative influence) of a reverse-engineered dynamic GRN model (Step 3) correspond to the parameters of a reference model (Step 1). We also use terms like matrix correlation to refer to $P_{\text {inf }}$ , as the calculation of $P_{\text {inf }}$ involves the comparison of the matrices representing the parameters of the reference (Step 1) and reverse-engineered (Step 3) dynamic GRN models respectively.

- Qualitative comparison, $Q_{\text {com }}$, refers to the extent a reverse-engineered GRN model corresponds with a reference model in terms of certain GRN features.

\subsubsection{Data fitting and predictive power}

Essentially, $P_{f i t}$ compares two sets of dynamic gene expression data, either predicted data with training data (here $P_{v e r}$, to verify a reverse-engineered model), or, predicted data with unseen test data (here $P_{\text {val }}$, to validate a reverse-engineered model). The general way to compute this score is the same and is defined as follows [28]:

$$
P_{f i t}=\frac{1}{(1+E)}
$$

where the error

$$
E=\frac{1}{p N} \sqrt{\sum_{t=1}^{p} \sum_{k=1}^{N}\left(X_{k t}-\hat{X}_{k t}\right)^{2}}
$$

where $X_{k t}$ denotes an experimentally observed gene product concentration level at time $t$ of gene $k$ and $\hat{X}_{k t}$ denotes a corresponding gene product concentration level estimated or predicted by the dynamic GRN model. $N$ denotes the number of genes in the GRN and $p$ the number of sampling points of the dynamic gene expression data.

$P_{f i t}$ assumes values form the unit interval, where 1 represents perfect accordance between the experimental data and the simulated or predicted data. However, care needs to be taken when interpreting $P_{f i t}$ values. In Table 8 , we indicate the model accuracy depending on the $P_{f i t}$ value. In data generated with our reference models, expression levels usually reach values of no more than 5 units. Thus $P_{f i t}$ values less than 0.9 indicate very poor quality of the reverse-engineered models, with dynamics that bear no relation to the original model. $P_{f i t}$ values between 0.95 and 0.99 indicate models with dynamics in much the same range as the original models, but the expression curves of the individual genes may exhibit different patterns.

$P_{f i t}$ values over 0.99 start to converge with the original model dynamics and a $P_{f i t}$ value of over 0.999 indicates a highly accurate accurate GRN model. 
Table 8 Meaning of $P_{\text {fit }}$ values

\begin{tabular}{cc}
\hline $\boldsymbol{P}_{\text {fit }}$ value & Max. RMS diff. \\
\hline 0.40 & 244.0 \\
\hline 0.75 & 76.9 \\
\hline 0.92 & 8.47 \\
\hline 0.97 & 2.34 \\
\hline 0.992 & 0.80 \\
\hline 0.997 & 0.42 \\
\hline 0.9992 & 0.13 \\
\hline 0.9997 & 0.067 \\
\hline
\end{tabular}

Interpretation of $P_{\text {fit }}\left(P_{\text {ver }}\right.$ and $\left.P_{\text {val }}\right)$ values: The typical maximum root mean square (RMS) difference (at a single time point) between the reverseengineered and reference GRN models, for the 5-gene models of case studies $A, B$, and $C$.

\subsubsection{Matrix correlation: Inferential power}

$P_{\text {inf }}$ is a deeper measure than $P_{f i t}$ and its variants $P_{v e r}$ and $P_{v a l}$, respectively [28]. It describes more fundamental properties (model parameters) of the process that generates data which is the basis for calculating $P_{f i t}$. In our study, we use $P_{\text {inf }}$ to compare the parameter matrix, $\mathbf{W}$, of reference models (used to generate the training and test data) with the parameter matrix, $\hat{\mathbf{W}}$ of reverseengineered models.

Inferential power, $P_{\text {inf }}$, is defined as follows:

$$
P_{\text {inf }}(\mathbf{W}, \hat{\mathbf{W}})=0.5(1+p(\mathbf{W}, \hat{\mathbf{W}}))
$$

where $p$ denotes the Pearson product-moment correlation coefficient between $\mathbf{W}$ and $\hat{\mathbf{W}}$.

$P_{\text {inf }}$ from Equation 13 assumes values from the unit interval; a value of 1 indicates an exact inference (estimation) of the model parameters. If there is an exact match between the parameter matrices of the reverseengineered and the reference GRN model, then the behavior of the two networks is identical under all conditions.

Since the three methods ANN, SS and GRLOT use different numbers of parameter values, we can only calculate $P_{\text {inf }}$ values for the cases where the method used for reverse-engineering equals the method used for generating the training data. In all other cases we make a qualitative estimation of $P_{\text {inf }}$.

\subsubsection{Qualitative comparison}

$Q_{\text {com }}$ refers to the similarity of a model with the true underlying system in terms of network features.

Primarily, network features refer to the network connectivity that captures the topology of a network and the connection "logic". However, in this study the network structure is given so that only network features such as the type of the effect (inhibitory or stimulatory) and its degree of influence need to be deduced or estimated. Network features are represented by the model's parameters. Since parameters of the three types of mathematical methods can not be directly compared to each other, we determine $Q_{\text {com }}$ by means of a qualitative comparison.

The qualitative analysis of the derived model parameters is performed according to the characteristics listed in Section 1.3.1. For example, when manually constructing the reference GRN models, we chose uniform parameter values to define degradation rates. Based on this and other characteristics described in Section 1.3.1, it is possible to estimate the accuracy of each model.

In this study, the characteristic uniform degradation rate is present in the reverse-engineered parameter matrices if all degradation rates within a matrix are within the interval [0.2,0.4]; constant signal propagation is present when the ratio between the weakest and the strongest signal is smaller than 2; asymmetric signal branching and asymmetric co-regulation is present when there is more than a $20 \%$ difference between the signals coming to or arising from the system variables. Finally, positive feedback and negative feedback are described in the dependencies between the variables X3 and X2, and $\mathrm{X} 5$ and X2 respectively.

\subsection{A note on the technical infrastructure and computational complexity of the experiments}

To perform the GRN reverse-engineering experiments, we developed a module in Narrator [49] that provided an automatic interface to the Condor [50] distributed, high-performance computing system. This allowed computing tasks defined by Narrator to be automatically placed into the Condor scheduling queue. We used two pools of Condor computing clusters that were dedicated to our experiments: Pool 1 consisted of 23 Fujitsu Siemens E600 machines, each with a single Pentium $4 \mathrm{HT}$ $3.06 \mathrm{GHz}$ processor and $1 \mathrm{~GB}$ RAM; Pool 2 consisted of 10 Dell Optiplex GX 620 machines, each with a single Pentium $4 \mathrm{HT} 3.4 \mathrm{GHz}$ processor and $1 \mathrm{~GB}$ RAM. A typical evolutionary optimization would take about 35 minutes for the molecular models and 150 minutes for the network models. With the Condor pools we could repeat these runs a number of times, as this helped overcome problems due to the search process getting stuck in a local minimum. In total, it therefore took about 27 hours of compute time to perform a single reverse-engineering experiment. (For a 5-gene network with a detailed data set: $5 \times 5$ molecular model runs +5 $\times 1$ network model run $=25 \times 35 \mathrm{~min}+5 \times 150 \mathrm{~min}=$ $875 \mathrm{~min}+750 \mathrm{~min}=1625 \mathrm{~min}$.)

\section{Acknowledgments}

The work in this paper was supported by EC grants DataMiningGrid IST FP6 004475 and QosCosGrid IST FP6 STREP 033883. Some of the experiments presented in this paper were carried out using the Grid'5000 experimental 
testbed, being developed under the INRIA ALADDIN development action with support from CNRS, RENATER and several Universities as well as other funding bodies (see https://www.grid5000.fr).

\section{Author details}

${ }^{1}$ Roche Diagnostics GmbH, Nonnenwald 2, 82372 Penzberg, Germany. ${ }^{2}$ University of Ulster, School of Biomedical Sciences, Cromore Road, Coleraine BT52 1SA, Co. Londonderry, UK.

\section{Authors contributions}

$\mathrm{JM}$ participated in conceiving and designing the experiments, performed some of the computational modelling, analyzed the results, and participated in drafting the manuscript. MS participated in conceiving and designing the experiments, performed some of the computational modelling, analyzed the results, and participated in drafting the manuscript. JM and MS contributed equally to this paper. WD participated in the design of the experiments and helped to draft the manuscript. All authors read and approved the final manuscript.

\section{Received: 21 April 2010 Accepted: 14 September 2010}

Published: 14 September 2010

\section{References}

1. Davidson E, Levin M: Gene regulatory networks. Proceedings of the National Academy of Sciences of the United States of America 2005, 102(14):4935.

2. Hasty J, McMillen D, Isaacs F, Collins JJ: Computational studies of gene regulatory networks: In numero molecular biology. Nature Reviews Genetics 2001, 2:268-279.

3. Spellman PT, Sherlock G, Zhang MQ, lyer VR, Anders K, Eisen MB, Brown PO, Botstein D, Futcher B: Comprehensive identification of cell cycleregulated genes of the yeast Saccharomyces cerevisiae by microarray hybridization. Molecular Biology of the Cell 1998, 9:3273-3297.

4. Arbeitman MN, Furlong EEM, Imam F, Johnson E, Null BH, Baker BS, Krasnow MA, Scott MP, Davis RW, White KP: Gene expression during the life cycle of Drosophila melanogaster. Science 2002, 297(5590):2270-2275.

5. Zvonic S, Ptitsyn AA, Conrad SA, Scott LK, Floyd ZE, Kilroy G, Wu X, Goh BC, Mynatt RL, Gimble JM: Characterization of peripheral circadian clocks in adipose tissues. Diabetes 2006, 55:962-970.

6. Ronen M, Rosenberg R, Shraiman BI, Alon U: Assigning numbers to the arrows: Parameterizing a gene regulation network by using accurate expression kinetics. Proceedings of the National Academy of Sciences of the United States of America 2002, 99(16):10555-10560.

7. Bar-Joseph Z: Analyzing time series gene expression data. Bioinformatics 2004, 20(16):2493-2503.

8. Kauffman S: The origins of order: Self-organization and selection in evolution Oxford University Press 1993.

9. Liang S, Fuhrman S, Somogyi R: Reveal, a general reverse engineering algorithm for inference of genetic network architectures. Proceedings of Pacific Symposium Biocomputing 1998, 18-29.

10. Akutsu T: Identification of genetic networks from a small number of gene expression patterns under the Boolean network model. Proceedings of Pacific Symposium Biocomputing 1999, 17-28.

11. Perrin BE, Ralaivola L, Mazurie A, Bottani S, Mallet J, d'Alche Buc F: Gene networks inference using dynamic Bayesian networks. Bioinformatics 2003, 19(2):138-148.

12. Cho KH, Choo SM, Jung SH, Kim JR, Choi HS, Kim J: Reverse engineering of gene regulatory networks. IET Systems Biology 2007, 1:149-163.

13. Stetter M, Schurmann B, Dejori M: Systems level modeling of gene regulatory networks. In Artificial intelligence methods and tools for systems biology. Edited by: Dubitzky W, Azuaje F. Springer, Netherlands; 2004:175-194.

14. Savageau MA: Biochemical Systems analysis: A study of function and design in molecular biology Addison-Wesley, Reading, Mass 1976.

15. Vohradsky J: Neural network model of gene expression. The FASEB Journal: Official publication of the Federation of American Societies for Experimental Biology 2001, 15(3):846-854.

16. Mendes $P$, Sha W, Ye K: Artificial gene networks for objective comparison of analysis algorithms. Bioinformatics 2003, 19(90002):122-129.

17. Quackenbush J: Computational analysis of microarray data. Nature Reviews Genetics 2001, 2(6):418-427.
18. knüpfer C, Dittrich P, Beckstein C: Artificial gene regulation: A data source for validation of reverse bioengineering. In Proceedings of the 6th German Workshop on Artificial Life 2004 Edited by: Schaub H, Detje F, Brggemann U 2004, 66-75.

19. Cantone I, Marucci L, lorio F, Ricci MA, Belcastro V, Bansal M, Santini S, di Bernardo M, di Bernardo D, Cosma MP: A yeast synthetic network for in vivo assessment of reverse-engineering and modeling approaches. Cell 2009, 137:172-181.

20. Herrera F, Lozano M, Verdegay JL: Tackling real-coded genetic algorithms: Operators and rools for behavioural analysis. Artificial Intelligence Review 1998, 12(4):265-319.

21. Hlavacek W, Savageau M: Rules for coupled expression of regulator and effector genes in inducible circuits. Journal of Molecular Biology 1996, 255:121-139.

22. Crampin EJ, Schnell S, McSharry PE: Mathematical and computational techniques to deduce complex biochemical reaction mechanisms. Progress in Biophysics and Molecular Biology 2004, 86:77-112.

23. Almeida JS, Voit EO: Neural-network-based parameter estimation in Ssystem models of biological networks. Genome Informatics 2003, 14:114-123.

24. Wildenhain J, Crampin EJ: Reconstructing gene regulatory networks: From random to scale-free connectivity. IEE Proceedings of Systems Biology 2006, 156(4):247-256.

25. Veitia R: A sigmoidal transcriptional response: cooperativity, synergy and dosage effects. Biological Reviews 2003, 78(01):149-170.

26. D'haeseleer P, Liang S, Somogyi R: Genetic network inference: From coexpression clustering to reverse engineering. Bioinformatics 2000, 16(8):707-726.

27. Spieth C, Streichert F, Speer N, Zell A: A memetic inference method for gene regulatory networks based on S-systems. Proceedings of the IEEE Congress on Evolutionary Computation 2004, 152-157.

28. Wessels $L$, van Someren $E$, Reinders M: A comparison of genetic network models. Proceedings of the Pacific Symposium on Biocomputing 2001, 508-519.

29. van Someren EP, Wessels LFA, Reinders MJT: Linear modeling of genetic networks from experimental data. Proceedings of the 8th International Conference on Intelligent Systems for Molecular Biology 2000, 55-366.

30. Yuan S, Li KC: Context-dependent clustering for dynamic cellular state modeling of microarray gene expression. Bioinformatics 2007, 23(22):3039-3047.

31. Yang Y, Kim J, Song E, E K, Oh M, BG K: Finding new pathway-specific regulators by clustering method using threshold standard deviation based on DNA chip data of Streptomyces coelicolor. Appl Microbiol Biotechnol 2008, 80:707-717.

32. Nam D, Yoon SH, Kim JF: Ensemble learning of genetic networks from time-series expression data. Bioinformatics 2007, 23(23):3225-3231.

33. Kimura S, Ide K, Kashihara A, Kano M, Hatakeyama M, Masui R, Nakagawa N, Yokoyama S, Kuramitsu S, Konagaya A: Inference of S-system models of genetic networks using a cooperative coevolutionary algorithm. Bioinformatics 2005, 21(7):1154-1163.

34. Guthke R, Moller U, Hoffmann M, Thies F, Topfer S: Dynamic network reconstruction from gene expression data applied to immune response during bacterial infection. Bioinformatics 2005, 21(8):1626-1634.

35. Milo R, Shen-Orr S, Itzkovitz S, Kashtan N, Chklovskii D, Alon U: Network motifs: Simple building blocks of complex networks. Science 2002, 298:824-827.

36. Lee TI, Rinaldi NJ, Robert F, Odom DT, Bar-Joseph Z, Gerber GK, Hannett NM, Harbison CT, Thompson CM, Simon I, Zeitlinger J, Jennings EG, Murray HL, Gordon DB, Ren B, Wyrick JJ, Tagne JB, Volkert TL, Fraenkel E, Gifford DK, Young RA: Transcriptional regulatory networks in Saccharomyces cerevisiae. Science 2002, 298(5594):799-804.

37. Noman $\mathrm{N}$, lba H: Reverse engineering genetic networks using evolutionary computations. Genome Informatics 2005, 16(2):205-214.

38. Iba H, Mimura A: Inference of a gene regulatory network by means of interactive evolutionary computing. Inf Sci Inf Comput Sci 2002, 145(34):225-236

39. Schwefel HP: Evolution and optimum seeking Wiley Interscience 1995.

40. Koza JR, David Andre, Bennett FH III, Keane M: Genetic programming 3: Darwinian invention and problem solving Morgan Kaufman 1999.

41. Michalewicz Z: Genetic algorithms + data structures = evolution programs Springer 1992. 
42. Aho T: Simulation tool for genetic regulatory networks Masters thesis Dept. of Information Technology, Tampere University of Technology, Finland 2003.

43. $V u T T$, Vohradsky J: Nonlinear differential equation model for quantification of transcriptional regulation applied to microarray data of Saccharomyces cerevisiae. Nucleic Acids Research 2007, 35:279-287.

44. $\mathrm{Vu} T \mathrm{~T}$, Vohradsky J: Inference of active transcriptional networks by integration of gene expression kinetics modeling and multisource data. Genomics 2009, 93(5):426-433.

45. Maki $Y$, Ueda T, Okamoto M, Uematsu N, Inamura K, Uchida K, Takahashi $Y$, Eguchi $Y$ : Inference of genetic network using the expression profile time course data of mouse P19 cells. Genome Informatics 2002, 13:382-383.

46. Kimura S, Hatakeyama M, Konagaya A: Inference of S-system models of genetic networks using a genetic local search. In Proceedings of the 2003 Congress on Evolutionary Computation. Edited by: Sarker R, Reynolds R, Abbass H, Tan KC, McKay B, Essam D, Gedeon T. IEEE Press; 2003:631-638.

47. Eshelman LJ, Schaffer JD: Real-coded genetic algorithms and interval schemata. In Foundations of genetic algorithms 2. Edited by: Whitley LD. Morgan Kaufmann Publishers; 1993:

48. Bolze R, Cappello F, Caron E, Dayd'e M, Desprez F, Jeannot E, J'egou Y, Lanteri S, Leduc J, Melab N, Mornet G, Namyst R, Primet P, Quetier B, Richard O, Talbi EG, Touche I: Grid'5000: A large scale and highly reconfigurable experimental grid testbed. The International Journal of High Performance Computing Applications 2006, 20(4):481-494.

49. Mandel JJ, Fuss H, Palfreyman NM, Dubitzky W: Modeling biochemical transformation processes and information processing with Narrator. BMC Bioinformatics 2007, 8(103)

50. Thain D, Tannenbaum T, Livny M: Distributed computing in practice: The Condor experience. Concurrency - Practice and Experience 2005, 17(24):323-356.

doi:10.1186/1471-2105-11-459

Cite this article as: Swain et al: Comparative study of three commonly used continuous deterministic methods for modeling gene regulation networks. BMC Bioinformatics 2010 11:459.

\section{Submit your next manuscript to BioMed Central and take full advantage of:}

- Convenient online submission

- Thorough peer review

- No space constraints or color figure charges

- Immediate publication on acceptance

- Inclusion in PubMed, CAS, Scopus and Google Scholar

- Research which is freely available for redistribution

Submit your manuscript at www.biomedcentral com/submit 